\title{
1 Retinal factors of visual sensitivity in the human fovea
}

2 Niklas Domdei, Jenny L. Reiniger, Frank G. Holz, Wolf Harmening

Department of Ophthalmology, University of Bonn, Bonn, Germany

4

\section{Abstract}

Humans direct their gaze towards visual objects of interest such that the retinal images of fixated objects fall onto the fovea, a small anatomically and physiologically specialized region of the retina displaying highest visual fidelity. One striking anatomical feature of the fovea is its non-uniform cellular topography, with a steep decline of cone photoreceptor density and outer segment length with increasing distance from its center. We here assessed in how far the specific cellular organization of the foveola is reflected in visual function. Increment sensitivity to small spot visual stimuli ( $1 \times 1$ arcmin, $543 \mathrm{~nm}$ light) was recorded psychophysically in 4 human participants at 17 locations placed concentric within a 0.2-degree diameter around the preferred retinal locus of fixation with adaptive optics scanning laser ophthalmoscopy based microstimulation. While cone density as well as maximum outer segment length differed significantly among the four tested participants, the range of observed threshold was similar, yielding an average increment threshold of $3.3 \pm 0.2 \log 10$ photons at the cornea. Thresholds were correlated with retinal eccentricity, as well as cone density and outer segment length. Biophysical simulation allowed to develop a model of foveal sensitivity based on these parameters, explaining at least $37 \%$ of the observed threshold variability. Based on high reproducibility in replicate testing, the residual variability is assumed to be caused by individual cone and bipolar cell weighting at the specific target locations. 


\section{Introduction}

The presence of a fovea in the retina of some birds, fish, reptiles and primates like humans (Slonaker, 1897), provides an outstanding opportunity to study the linkage between a sensory system's cellular architecture and its function. In humans, the fovea features several morphologic specializations as a result of a complex series of events that are initiated during gestation and completed in childhood (Yuodelis and Hendrickson, 1986; Zhang et al., 2020). The network of retinal blood vessels organizes itself around an avascular zone concentric within the fovea (Provis et al., 2013) and the foveal pit emerges as post-receptoral neurons migrate laterally. As a consequence, the foveola, the central 0.6 degree diameter of the fovea (Cuenca et al., 2020), is completely free of overlying neural tissue, favoring undisturbed light catch. Cone photoreceptors migrate inwards to form a lattice of tightly packed receptors at their smallest diameter found anywhere in the retina (Curcio et al., 1990), and simultaneously, the pigmentladen cone outer segments elongate (Yuodelis and Hendrickson, 1986), leading to a peak in optical density at the foveola (Elsner et al., 1993; Marcos et al., 1997). Psychophysically, photopic light sensitivity drops rapidly outside of the foveola (Choi et al., 2016).

At close inspection, the topography and density of cone photoreceptors within the foveola is highly variable between individuals (Wang et al., 2019) and it is not known, to what extent the individual cellular mosaic and the exact foveal topography is related to visual sensitivity. In patients with inherited retinal degeneration, for example, light sensitivity in the fovea was similar to a healthy control group and showed declined sensitivity only when peak cone density was reduced to less than $60 \%$ of the average (Foote et al., 2018). Sensitivity to light is assumed to be conveyed primarily by parasol ganglion cells (Takeshita et al., 2017), supported by the observation that spatial summation is correlated with the receptive field size of parasol ganglion cells (Volbrecht et al., 2000). In the foveola, however, the spatial summation area is less than 5 $\operatorname{arcmin}^{2}$, between the dendritic field size of parasol and midget ganglion cells (Tuten et al., 
2018). Thus, a visual stimulus smaller than the spatial summation area might reveal an otherwise obscured relationship between the peaked topography of the detector array in the foveal center and its visual sensitivity.

Concomitant to the peaking spatial sampling capacity at the fovea, motor circuits of the brain stem generate eye and head movements to bring the retinal images of objects of interest detected in the periphery into the foveola (Goldberg and Walker, 2013; Poletti et al., 2013), where they fall on a distinct bouquet of only a handful cones, the preferred retinal location of fixation (PRL or "optimal locus") (Nachmias, 1959; Steinman, 1965; Putnam et al., 2005). This subset of cones at the PRL may have functional preeminence, as fixational eye movements recenter the object of interest on this location (Pritchard, 1961). However, previous studies showed that the PRL does not colocalize with structural features such as peak cone density (Putnam et al., 2005) or the center of the foveal pit volume (Wilk et al., 2017a).

With recent optical tools, the mosaic of even the smallest cone photoreceptors in the foveola can now be resolved in the living human eye and visual function such as visual sensitivity can be simultaneously probed psychophysically with cellular precision (Harmening et al., 2014). In a first attempt to better understand how the specific mosaic of cone photoreceptors within the foveal center has functional consequences to vision, we here use this experimental access to study the direct relationship between the cellular makeup of the foveal center and sensitivity to light in four human participants. 


\section{Methods}

In four human participants ( 1 female, 3 males; $35 \pm 6$ years), the cellular topography of the fovea, light sensitivity to cone-sized stimuli, and the preferred retinal locus of fixation where mapped with cellular precision using an adaptive optics scanning laser ophthalmoscope (AOSLO) microstimulator. The participants were three of the authors $(P 1=N D, P 2=J L R, P 3=$ WMH), and one lab member (P4). Pupil dilation and cycloplegia were induced by instilling one drop of $1 \%$ Tropicamide 15 minutes before the beginning of an experimental session. For each participant, a custom dental impression (bite bar) was used to immobilize and control the position of the head during imaging and stimulation. Written informed consent was obtained from each participant and all experimental procedures adhered to the tenets of the Declaration of Helsinki, in accordance with the guidelines of the independent ethics committee of the medical faculty at the Rheinische Friedrich-Wilhelms-Universität of Bonn, Germany.

\section{Adaptive optics scanning laser ophthalmoscope microstimulator}

The central $\sim 1$ degree of the right eye of each participant was imaged and targeted test sites were simultaneously stimulated with a custom multiwavelength AOSLO (for details of the system see (Poonja et al., 2005; Grieve et al., 2006; Domdei et al., 2018, 2019)). In brief, the output of a supercontinuum light source (SuperK Extreme EXR-15, NKT Photonics, Birkerød, Denmark) was split into two light channels by serial dichroic and bandpass filtering: one near infrared (IR) light channel was used for imaging and wavefront sensing (840 $\pm 12 \mathrm{~nm}$, FF01840/12-25, Semrock, Rochester, USA), and a visible green light channel for microstimulation (543 \pm 22 nm, FF01-543/22-25, Semrock). Adaptive optics correction, run in closed loop operation at about $25 \mathrm{~Hz}$, consisted of a Shack-Hartmann wavefront sensor (SHSCam AR-S150-GE, Optocraft GmbH, Erlangen, Germany) and a magnetic 97-actuator deformable mirror (DM97-08, ALPAO, Montbonnot-Saint-Martin, France) placed at a pupil conjugate. Imaging and stimulation beams, traversing the system coaxilly, were point-scanned across the retina, 
spanning a square field of $0.85 \times 0.85$ degrees of visual angle. The light reflected from the retina was detected in photomultiplier tubes (PMT, Photosensor module H7422-40 and -50 Hamamatsu Photonics, Hamamatsu, Japan) individually for each channel, placed behind a confocal pinhole (pinhole diameter $=20 \mu \mathrm{m}$, equaling $0.47(840 \mathrm{~nm})$ and $0.71(543 \mathrm{~nm})$ Airy disk diameters). The PMT signals were continuously sampled by an FPGA board (ML506, Xilinx, San Jose, USA), producing video frames with $512 \times 512$ pixel (spatial resolution $=0.1$ arcmin of visual angle per pixel) at $30 \mathrm{~Hz}$ by combining the PMT's voltage output with the positional data of the scanners. To increase dynamic range and contrast, the $543 \mathrm{~nm}$ stimulus light was modulated by two cascaded acousto-optic modulators (Domdei et al., 2018). The stimulation light was attenuated by 3 log units using a neutral density filter before coupling light into the AOMs, ensuring an optimal operating range of the AOMs. The combined effects of chromatic aberrations of the human eye were minimized as follows: Iongitudinal chromatic aberration, LCA, was compensated by a static relative vergence difference of 1 diopter between the $840 \mathrm{~nm}$ and $543 \mathrm{~nm}$ light channel (Atchison and Smith, 2005), and an individual adjustment of defocus of the DM for each eye, prioritizing image quality in the visible channel. Transverse chromatic offsets were compensated dynamically with every stimulus presentation by lateral stimulus location offsets driven by correction signals from Purkinje-based pupil monitoring (Harmening et al., 2012; Domdei et al., 2019).

\section{Cone density maps and cone density centroid}

Continuous maps of cone photoreceptor density were generated by recording a 10 second AOSLO video with an imaging wavelength of $543 \mathrm{~nm}$ (P1 and P2) or $788 \mathrm{~nm}$ (P3 and P4) while the participant was asked to fixate on a small flashing target in the center of the imaging raster. High signal-to-noise ratio images were then generated by strip-wise image registration and averaging of individual frames of those videos, while manually excluding single frames with failed registration. In these normalized images, one human grader marked the location of each 
cone, assisted by convolutional neural network custom software (Cunefare et al., 2017; Reiniger et al., 2019). Matlab's Voronoi function (voronoiDiagram, Mathworks, Inc., Natick, MA, USA) was used to compute each cone's area. Cone density at each pixel of this image was then calculated by first identifying the nearest 150 cones around each pixel in the image. Cone density was then defined by dividing the number of cones by their summed area. The cone density centroid (CDC) was the retinal location found as the weighted centroid (Matlab function regionprops(Image, 'weightedcentroid')) of the area containing the highest $20 \%$ cone density values.

\section{Determination of cone outer segment length}

Spectral-domain optical coherence tomography (OCT) images were acquired for all 4 participants in a $5 \times 15$ degree field, centered on the fovea with a B-scan spacing of $11 \mu \mathrm{m}$ (HRA-OCT Spectralis, Heidelberg Engineering, Heidelberg, Germany). For further processing, the central $45 \mathrm{~B}$-scans around the foveal pit were selected. Cone outer segment (OS) length was defined as the space between visible bands 2 and 3 in each B-scan, thus is the space between the ellipsoid zone and the interdigitation zone, respectively (Spaide and Curcio, 2011). In a first step, these two bands were segmented by manually adjusting a brightness-based automated detection algorithm (Fig. 5A). Because the retinal pigment epithelium is thought to have a relatively uniform thickness and flat layout, a two-dimensional area fit across all B-scans for the third band was computed, reducing artifacts of the individual band marking. The width of both bands was modelled with a 1D gaussian profile, centered on the band marking. The OS length was defined as the linear space between the steepest parts of the second band's declining and the third band's rising slope (see also Spaide and Curcio (2011)). In the final step, the two-dimensional OS length map was smoothed along the orthogonal B-scan axis with a Savitzky-Golay filter to remove residual artifacts of individual band markings between the single B-scans and then rescaled to obtain equal increment per pixel values in all directions. These 2D 
maps of OS length were registered with retinal AOSLO imagery by centering their maximum value at the CDC.

\section{PRL determination}

The preferred retinal locus of fixation (PRL) was determined by recording the exact retinal location at which a small, flashing visual stimulus landed during attempted fixation. The fixation stimulus was created by modulating the imaging channel of the AOSLO to turn off in a central region of the raster, creating a small visible black square, $1.6 \times 1.6$ arcmin nominal size, shown against the visible $840 \mathrm{~nm}, 51 \times 51$ arcmin scanning background. The stimulus flashed continuously at $3 \mathrm{~Hz}$ and its retinal landing positions were recorded in three consecutive 10 second AOSLO videos. Retinal fixation locations were then found by, (1) automatically registering each frame strip-by-strip to a common reference frame of a single video (Arathorn et al., 2007), (2) manual deletion of incorrectly registered frames (usually due to microsaccades, insufficient image quality or eye blinks), (3) tabulating all stimulus locations within the remaining, co-registered frames (Fig. 1A). The final PRL estimate for each eye was the median stimulus location across all three videos, equaling on average 387 frames (Fig 1B). This location was defined as 0 degree eccentricity.

\section{Increment sensitivity thresholds}

After PRL determination, participants underwent small spot sensitivity testing at multiple sites within their foveola, with the PRL as a spatial anchor at the center of the test locations. Sixteen additional test locations were selected manually around the PRL, close to the intersections of two concentric perimeters (spaced 6 and 12 arcmin) around the PRL with the horizontal, vertical, and diagonal meridians (Fig. 2A and B). At each of the 17 test locations, sensitivity thresholds were determined as the median of 3-5 repeat measurements per location. In each 
experimental run, three out of the 17 locations that were oriented closely along a vertical axis were tested as a group, with the individual locations tested pseudo-randomly interleaved within the group. This approach minimized participant fatigue, allowed for more regular breaks between runs, and also minimized background intensity changes that are present towards the horizontal edges of the imaging raster ( \pm 0.05 contrast change), because stimuli could be presented in the central part of the imaging raster at all times. Sensitivity thresholds at each test location were estimated with QUEST, an adaptive staircase method (Watson and Pelli, 1983), adjusting stimulus intensity following a Yes/No-test paradigm with logarithmic step sizes (KingSmith et al., 1994). Because of the visible IR imaging raster, stimuli were presented against a reddish background (about $5 \mathrm{~cd} / \mathrm{m}^{2}$ photopic luminance), rendering our sensitivity testing paradigm to yield increment sensitivity thresholds (see also Harmening et al. (2014); Tuten et al. (2017)). The test stimulus was a green square with 7 pixels edge length in scanning raster coordinates. Considering a residual defocus of 0.03 D (Meadway and Sincich, 2018), the stimulus edge length given by the full width at half maximum was about 0.8 arcmin, or between $150 \%(\mathrm{P} 1)$ and $180 \%(\mathrm{P} 4)$ the diameter of the smallest cones. Due to the scanning nature of the AOSLO, where visual stimuli are rendered pixel-by-pixel while the illumination beam traverses the retina, the presentation time spanned $350 \mu \mathrm{sec}$ from the first to the last pixel of the stimulus. This equals a net illumination time per stimulus of $2.5 \mu \mathrm{sec}$. Stimulus presentation progression was self-paced and successful deliveries were accompanied by an auditory cue. Stimulus delivery was blocked if either the online stabilization algorithm or the eye tracking for TCO compensation failed, and the participant had to repeat the trial $(\sim 32.4 \%$ of all 5674 stimulus presentations). Threshold estimation was completed after 12 trials if the standard deviation (STD) value of QUEST was less or equal to 0.10 log arbitrary AOM voltage drive units (Fig. 2D). If STD was higher, the run was extended by additional trials until the STD criterion was met. The run was terminated and had to be repeated if the STD criterion was not met after 
18 trials. Runs were repeated until a minimum of three valid threshold estimates per test location were recorded.

Real-time image stabilization enabled retinal tracking and repeated stimulation of the targeted test sites (Arathorn et al., 2007). In a subsequent offline analysis, trials with suboptimal delivery (more than 0.6 arcmin deviance from median delivery location, equaling about 1.2 cone diameters) were excluded from further analysis. The non-linear swing speed of the resonant scanner produced a non-uniform IR background across the field, with increasing brightness close to the vertical edges of the field. For compensation, the individual trial intensity was corrected based on the position in the imaging raster and the resulting stimulus contrast, with compensation factors ranging between 0.95 and 1.00. After trial rejection and intensity correction, QUEST was computationally re-run with the updated stimulus intensities to recompute the final threshold estimate. Trial deletions on account of stabilization errors produced higher QUEST STDs. In a second step, thresholds with an STD higher than $0.15 \mathrm{log}$ a.u. were excluded from the following analysis. Sensitivity thresholds are finally computed and reported as the number of photons incident at the cornea at the threshold intensity (see next chapter).

\section{Conversion of arbitrary power units to number of photons at the cornea}

Before and after each experimental run, maximum output power of the AOSLO stimulation light channel was measured at maximum AOM drive voltage with a silicon photodiode and power meter (S130C and PM320E, Thorlabs GmbH, Bergkirchen, Germany) in the transmitted portion of the stimulation beam after a 90/10 (Transmisson/Reflection) beam splitter placed in the light delivery arm of the AOSLO. The average of these two measurements was used for actual stimulus power calculation of a given run. Typically, laser power fluctuated by less than $1 \%$ between measurements. With this, the QUEST threshold estimate (ThreshEst.), so far given in 
log10 arbitrary AOM drive units, could be converted into a number of photons at the cornea using formula (1):

$$
\text { Photons }_{\text {Thresh. }}=\frac{P_{\text {Max }} \cdot \text { Trans }_{\text {AOSLO }}}{E_{\text {Photon }}} \cdot t_{\text {Stim }} \cdot 10^{\text {ThreshEst }} .
$$

with $\mathrm{P}(\mathrm{Max})$ being the maximum AOM output power measured, Trans(AOSLO) being the relationship of maximum power fed into the AOSLO and the power detected at the eye's pupil position (determined earlier to be 0.065 ) and $t$ (Stim) being the stimulus duration during a single presentation (2.45 $\mu \mathrm{sec})$. $\mathrm{E}$ (Photon), the photon's energy, was calculated as:

$$
E_{\text {Photon }}=\frac{h \cdot c}{\lambda}
$$

with h, Planck's constant, c, the speed of light, and $\lambda$, stimulus light wavelength ( $543 \mathrm{~nm}$ ).

\section{Modelling of cone light capture and ISETBio}

To assess the impact of the exact stimulus location relative to the individual cone mosaic, a custom spatial model of quantal catch in the targeted cones was implemented in Matlab, similar to (Harmening et al., 2014). First, all stimulation trials were registered to a high-signal-to-noise image of the foveal center used to create the cone density map. Second, cone center locations were used to compute a complete Voronoi tessellation of the mosaic defining the inner segment area of the individual cones. Third, the actual absorption characteristic across this inner segment area was modelled by a two dimensional gaussian with a sigma value creating an aperture of 0.48 of the equivalent diameter (Macleod et al., 1992). The retinal stimulus was a two-dimensional convolution of the initial 7-by-7 AOSLO raster pixel stimulus and a diffraction limited point spread function with a residual defocus of 0.03 D (Meadway and Sincich, 2018). In the final step, the convolved stimulus was multiplied by the cone absorption matrix to arrive at 
the amount of stimulus light that was absorbed by each cone. The reported value of percent of light per cone is based on the total light distribution given by the convolved stimulus matrix.

But this model lacked a more generalized testing of the impact on thresholds by variations in the OS length or cone density. To test the hypothesis that the cone's biophysical properties such as cone density, cone diameter and cone outer segment length had an impact on sensitivity thresholds, the Image Systems Engineering Toolbox for Biology (ISETBio) for Matlab (Cottaris et al., 2019, 2020), was used. This computational-observer model simulates the cone photoreceptor isomerizations and photo currents based on physiological constraints. To set up ISETBio, the following parameters were used: the 'scene' was a $511 \times 511$ pixel image with the central $7 \times 7$ pixel containing the stimulus. The field of view was set to 0.85 degree and the background luminance set to $0.1 \mathrm{~cd} / \mathrm{m}^{2}$. The wavefront was set to be diffraction limited, with a varying residual defocus of $0.01,0.03$, and 0.05 diopters and zero LCA. The hexagonal mosaic function was used with a custom cone spacing and optical density. The optical density was calculated as the product of the OS length and an average absorbance of $0.014 \mu \mathrm{m}^{-1}(\mathrm{~L}-$-cones $=$ $0.013 \pm 0.002 \mu \mathrm{m}-1$ and M-cones $=0.015 \pm 0.004 \mu \mathrm{m}$, (Bowmaker et al., 1978)). Each condition (varying cone density, optical density and both coupled) was repeated 10 times taking simulated neural noise of the photoreceptor cells into account. To model different stimulus positions relative to the mosaic, the stimulus was shifted in steps of 0.6 arcsec (pixelwise at a magnification of 10). The test site's cone class composition was controlled by adjusting the Land M-cone spatial density parameter for each cone class and carefully checking the center surround configuration of the generated mosaic. These two simulations were both run with a residual defocus of 0.03 and repeated 33 times per condition. For Figure 10, the average of these data sets was plotted with the error bars reflecting one standard deviation. The slopes found by the ISETBio model for each parameter were used to calculate a factor specific offset $\Delta T_{i}$ from the average threshold for each of the test sites:

$$
\Delta T_{i}=\left(X_{i}-\bar{X}_{i}\right) \cdot m_{i}
$$


With $X_{i}$ being the test site's value of the factor $i$ (e.g. OS length), $\bar{X}$ the factor's average (31.39 $\mu m)$ and $m_{i}$ the factor specific slope. The sum of all three $\Delta X_{i}$ values yielded the test site's threshold offset from the average threshold. This way it was possible to compare the observed thresholds with an expected threshold based on the models without knowing the intercept of the factor specific function.

\section{Results}

Retinal topography and the $P R L$

With AOSLO imaging, the foveal mosaics of all four observers could be resolved, and all cone photoreceptors could be identified and marked to create continuous topographical maps of cone density. Participant numbering, P1-P4, was ordered for all following analysis according to their peak cone density values $\left(13733,15230,18023\right.$, and 18406 cones/degree ${ }^{2}$ for $P 1, P 2, P 3$, and $\mathrm{P} 4$ respectively). At the location of the cone density centroid (CDC, see Methods) the density values were slightly lower P1: 13460, P2: 15199, P3: 16956, P4: 16914 cones/deg². Cone density dropped rapidly with increasing eccentricity. Across subjects, cone density dropped on average to $66 \%$ of the peak cone density at a distance of about 20 arcmin from the CDC. However, there were marked differences in topographical profiles between subjects. For instance, $\mathrm{P} 1$ had a rather plateau like cone density distribution, while P4 showed a steep decline with a two-pronged cone density profile. The other participants had a similar slope in cone density profiles.

The two-dimensional map of cone outer segment (OS) length as determined by high-resolution OCT imaging was found to show a similar topography as cone density in each eye. $\mathrm{P} 1$, the participant with the lowest peak cone density, also had the lowest maximum OS length of 28 $\mu \mathrm{m}$. For P2, P3, and P4 the maximum OS length was 28,32 , and $40 \mu \mathrm{m}$, respectively. The 
range of OS length was $3 \mu \mathrm{m}$ ( 25 to $28 \mu \mathrm{m}$ ) for $\mathrm{P} 1,4 \mu \mathrm{m}(28$ to $32 \mu \mathrm{m})$ for $\mathrm{P} 2,4 \mu \mathrm{m}$ (29 to 33 $\mu \mathrm{m})$ for $\mathrm{P} 3$, and $7 \mu \mathrm{m}$ (32 to $40 \mu \mathrm{m})$ for P4 within the central 12 arcmin radius around the CDC. The PRL was found to be offset from the CDC and the location of highest OS length in all eyes. It was shifted away from a nasal-inferior quadrant of the retina with respect to the CDC with an average offset of about 3 arcmin. For each participant, the offset, as well as the angle, were different, ranging from 1 arcmin and 15 degree (P1) to 3 arcmin and 178 degree (P4) with a good repeatability of about 1 arcmin (Fig. 3A). PRL ellipses were found to be small in all participants (BCEA: P1 = 50; P2 = 67; P3 = 16; P4 = $30 \operatorname{arcmin}^{2}$ ), indicative of high fixation stability, a prerequisite for the following cone-targeted sensitivity testing.

\section{Small spot sensitivity}

Small spot sensitivity thresholds across subjects were found to lie between 2.97 and $3.67 \log 10$ photons, spanning an overall range of $0.7 \log 10$ photons. The combined median of all thresholds across retinal locations and participants was $3.25 \log 10$ photons at the cornea and the average $3.27 \pm 0.16 \log 10$ photons. While the range of thresholds across the four participants was similar $(3.04$ to $3.59,2.99$ to $3.53,3.02$ to 3.67 , and 2.97 to $3.51 \log 10$ photons for $\mathrm{P} 1, \mathrm{P} 2, \mathrm{P} 3$, and $\mathrm{P} 4$ respectively; see Figure 3B), there was a continuous shift of the median, 1st, and 3rd quartile of thresholds from P1 to P4. The median thresholds shifted by $0.2 \log 10$ photons from 3.35 for $\mathrm{P} 1$ towards 3.15 for $\mathrm{P} 4$ (3.24 for $\mathrm{P} 2$ and $\mathrm{P} 3$ ). For $\mathrm{P} 2$ and $\mathrm{P} 3$ the 1 st and 3rd quartile of thresholds were lower than for $\mathrm{P} 1$, but higher than for $\mathrm{P} 4$, this difference was not significant (Q1: 3.23, 3.20, 3.20, and 3.10; Q3: 3.43, 3.38, 3.34, and 3.25). The repeatability for each test site, computed across three to five reruns, was high, with an average standard deviation of $0.06 \pm 0.03 \log 10$ photons (Fig. 3C). The standard deviation across reruns was higher for $\mathrm{P} 3$ (average $=0.08 \log 10$ photons) compared to the other three participants $(0.05$ $\log 10$ photons). 


\section{Correlation between retinal structure and function}

To bring sensitivity thresholds into spatial correspondence with the structural data and fixation behavior, averaged retinal images derived from all four independent analysis steps (cone density maps, OS length map, PRL determination, and microstimulation target sites) were carefully aligned with each other (Fig. 4 and 5), to allow a pointwise and cellular resolved comparison between retinal structure and function.

As a first observation, for none of the participants, the target site with the lowest threshold and therefore highest sensitivity was at the PRL or fell within the fixation ellipse $(5.7,6.5,6.5$, and 6.0 arcmin distance). The average distance between the test site with lowest threshold and highest cone density was 7.3 arcmin. In P1 and P2 the CDC was closer to the target site with the lowest threshold than the PRL (CDC distances: 4.5, 3.3, 7.8, 9.8 arcmin). The range of cone densities at the test sites differed clearly within and between participants, with a difference from minimum to maximum of 2300 cones $/ \mathrm{deg}^{2}$ (11215 to 13198 cones $/ \mathrm{deg}^{2}$ ) for $\mathrm{P} 1,3400$ cones/deg${ }^{2}$ (12564 to 14971 cones/deg ${ }^{2}$ ) for P2, 6100 cones/deg ${ }^{2}$ (11786 to 17881 cones/deg ${ }^{2}$ ) for P3, and 3800 cones/deg² (14364 to 18132 cones/deg $)$ for P4. While the highest cone density of $\mathrm{P} 4$ was about one third higher than the peak cone density of $\mathrm{P} 1$, sensitivity thresholds were similar at those locations. However, a general trend was noticeable that most of the sensitivity thresholds observed in P1 were higher than most of the thresholds of P4.

For further correlation analysis, we used the median threshold of repeated threshold estimations at the same test location. The difference between individual sensitivity thresholds and their median can be seen comparing Figure $6 \mathrm{~A}$ with $6 \mathrm{~B}$. While there was no general significant correlation between the distance from PRL and sensitivity thresholds (Fig. 6B), the lowest thresholds were observed at 6 arcmin PRL distance for all participants, and the median threshold at this eccentricity was almost identical (P1 and P2) or even lower (P3 and P4) than the median threshold at the PRL. For the 12 arcmin eccentricity, we observed a similar or higher 
median threshold compared to the median PRL threshold. In P1 we observed an extremely high threshold at one location that was $0.3 \log 10$ photons higher than the second highest threshold.

Plotting the individual thresholds as a function of the test site's cone density revealed a tendency towards lower thresholds for higher cone densities (Fig. 6C), with a correlation coefficient of $\rho=-$ 0.09 for $\mathrm{P} 1, \rho=-0.49$ for $\mathrm{P} 2, \rho=-0.32$ for $\mathrm{P} 3$ and $\rho=-0.52$ for $\mathrm{P} 4$. The slope showed $\mathrm{a}$ tendency to be steeper for higher correlation coefficients: $m=-0.02,-0.06,-0.03$, and -0.07 $\log 10$ photons per $10^{3}$ cones/degree ${ }^{2}$ for $\mathrm{P} 1, \mathrm{P} 2, \mathrm{P} 3$, and $\mathrm{P} 4$, respectively).

Correlating thresholds with foveal cone OS length revealed a similar trend: thresholds were generally lower at sites with higher OS length (Fig. 6D). Correlation coefficients were $\rho=-0.18$ for $\mathrm{P} 1, \rho=-0.45$ for $\mathrm{P} 2, \rho=-0.31$ for $\mathrm{P} 3$ and $\rho=-0.52$ for $\mathrm{P} 4$. The slope was almost the same across the four participants $(\mathrm{m}=-0.03,-0.05,-0.04$, and $-0.04 \log 10$ photons per micron for $\mathrm{P} 1$, P2, P3, and P4, respectively).

When data was pooled across subjects, the correlation between the thresholds and the distance from the PRL was given by a slope of $0.65 \log 10$ photons per degree $(\rho=0.27$, Fig. $7 A)$. The observed decrease of thresholds at 6 arcmin distance from the PRL remained in the combined dataset. Thresholds as a function of cone density had a negative slope of $-0.04 \log 10$ photons per $10^{3}$ cones $/$ degree $^{2}(\rho=-0.45$, Fig. 7B). The correlation between thresholds and OS length indicated an average decrease of thresholds by $-0.02 \log 10$ photons per micron in the pooled data set $(\rho=-0.43$, Fig. $7 C)$.

Because the individual factors, distance from PRL, cone density and OS length are all highly significantly correlated with each other ( $p<0.001$, data not shown), a physiological model of cone light capture (ISETBio) was employed to model the impact of two of the three factors independently. 
Modelling the impact of cone density, OS length and distance from PRL on sensitivity

The first hypothesis tested with ISETBio modelling was if the highly significant correlation between detection thresholds and cone density could be caused by spatial summation effects. The stimulus used in sensitivity testing was about 1.5 times the average foveal cone diameter (see methods) and therefore the average distance to the surrounding neighbors could have played an important role. Using the cone spacing according to the individual cone densities of our participants, the model showed a correlation of about $-0.01 \log 10$ photons per $10^{3}$ cones/degree ${ }^{2}$, roughly four times lower than the observation in the behavioral data (Fig. 8A). ISETBio was also used to test the influence of outer segment length and associated optical density on sensitivity. The outer segment length was estimated for each participant from foveal OCT B-scans (Fig. 5), with a range of $25 \mu \mathrm{m}$ to $40 \mu \mathrm{m}$, and fed into the model. A strong impact of outer segment lengths on thresholds was predicted $(\rho=-0.99)$, with a slope of $-0.008 \log 10$ photons per $\mu \mathrm{m}$ OS length (Fig. 8B).

In the following step, the slopes derived for $0.03 \mathrm{D}$ residual defocus (Meadway and Sincich, 2018) were used to remove the estimated proportional influence of cone density and OS length from the observed thresholds. The corrected thresholds were supposed to vary only due to retinal eccentricity. These thresholds were then used to compute the location of the sensitivity centroid (SC) for each participant (Fig. 3) by finding the retinal coordinate yielding the highest value of $\rho$ for corrected thresholds against distance from this coordinate (Fig. $8 \mathrm{C}$ ). The SC was always offset from the PRL $(11.7,3.8,4.8$, and 7.5 arcmin distance) or $\operatorname{CDC}(13.1,2.2,6.8$, and 3.7 arcmin distance). When all rescaled thresholds were plotted as a function of distance to the SC, a linear fit with a slope of $0.69 \log 10$ photons per degree eccentricity or $0.012 \log 10$ photons per $\operatorname{arcmin}(\rho=0.46)$ emerged.

Finally, we applied these three slopes to model the expected shift from the average observed threshold, due to the test site's cone density, OS length and SC distance (Fig. 9). Because the $y$-intercept $b$ was unknown, the expected shift was calculated for each of the three factors based 
on the difference from its average. The estimated thresholds were best fitted by a linear regression with a slope of $1.03\left(\rho=0.38, R^{2}=0.37\right)$, confirming that the interaction of cone spacing, OS length and eccentricity yielded a good predictor for sensitivity thresholds. Given a residual variability of $\pm 0.15 \log 10$ photons from the prediction, we also looked at the variability that is possibly due to the exact stimulus position on the cone mosaic. As our stimulus was roughly 1.5 foveal cone diameters in size, the number of isomerizations elicited could have depended on the stimulus being centered on a single cone or in the middle between three cones.

\section{Modelling the impact of stimulus position and cone class composition}

To test the hypothesis that the exact placement of the test stimulus relative to the cone mosaic bears on sensitivity at that site, photon catch of each cone for the average stimulus location during each threshold experiment was modelled. The exact cone locations relative to the stimulus location were determined by registering the experiment's video data with the high signal-to-noise image used for cone annotation. An example of the result of that model is shown in Fig. 10A for two different cases. The first case shows two different target sites in the retina of P2. At both target sites, the actual stimulus location on the cone mosaic was similar, but thresholds differed significantly by $0.36 \log 10$ photons $(p=0.03$, Mann-Whitney U-test, $n=4)$. In the second example from $\mathrm{P} 4$, the stimulus placement differed, the first one was centered on a single cone while the second one was placed in the middle of three cones. For this case we found similar sensitivity thresholds, with a non-significant difference $(p=0.49$, Mann-Whitney Utest, $n=4)$. When all thresholds were plotted against light catch in the nearest cone, no significant correlation emerged (Fig. 10B). The ISETBio model, creating a generalized perfect hexagonal retinal mosaic, supported this observation, when shifting the stimulus systematically from a cone-centered position to a position in the middle between cones (Fig. 10C). For such shifts, the resulting change given by the number of isomerizations was 0.1 in log 10 space. 
422 There was no difference if the center cone was a L- or M-cone. Such small changes could not

423 explain the observed variability of $\pm 0.3 \log 10$ photons, but could be one reason for the observed

424 intra-run variability of $0.06 \log 10$ photons due to small stimulus displacements caused by

425 residual errors of the real-time stabilization (Fig. 1C).

426 Another reason causing such variability could be the composition of cone classes at the

427 stimulus location. Again, ISETBio modelling showed only minor changes in the sum of isomerisation for different cone mosaic compositions (Fig. 10D). The maximum difference was $0.02 \log 10$ isomerisations for a stimulus location consisting of only L-cones versus only Mcones. As the actual composition of the cone mosaic in our four participants was unknown, this model did not contain any specific L-M-cone interactions and only used the cone class specific spectral sensitivity.

\section{Discussion}

The human fovea is the result of morphologic specializations culminating in tight photoreceptor packing and elongation of the photopigment containing outer segments (OS). Eye movements constantly align fixated objects of interest to a distinct group of cones in the fovea, the preferred retinal locus of fixation (PRL). The PRL and the retinal location where anatomical features peak are known to be offset from each other. By using an adaptive optics scanning laser ophthalmoscope (AOSLO) as high-resolution imaging and microstimulation platform, the functional profile of the foveola was further investigated. In four observers, increment sensitivity thresholds at a number of target sites around the PRL were correlated with retinal factors such as cone density, photoreceptor OS length, and the distance from the PRL. We generally found a plateau-like sensitivity profile within the central 6-arcmin radius around the PRL with a declined sensitivity at about 12 arcmin eccentricity, resulting in a much flatter slope than the anatomical topography of cone density and OS length changes in the same region. However, about $37 \%$ of the variability in thresholds could be explained by a combined model of the impact of cone 
density, OS length and distance from the PRL on thresholds, indicating that the exact makeup of the foveal cone mosaic and fixation behavior are indeed factors of visual function at cellular scale.

The average threshold for detecting a cone-sized visual stimulus against a dim red background $\left(5 \mathrm{~cd} / \mathrm{m}^{2}\right.$ at $\left.840 \mathrm{~nm}\right)$ was $3.27 \pm 0.16 \log 10$ photons at the cornea (Fig. 4). While rods enable the detection of individual photons (Sakitt, 1972; Tinsley et al., 2016), the absolute detection threshold at the fovea, and therefore based on cone vision only, was reported to be about 2.8 log10 photons (Marriott, 1963; Geisler and Davila, 1985) or $2.3 \log 10$ photons (Koenig and Hofer, 2011). Because the here reported thresholds are increments against the IR scanning background, the cones are adapted to the background resulting in elevated thresholds. The best comparison is given by a previous AOSLO microstimulation study on the foveal cone summation area, reporting an average increment threshold of $4.4 \log 10$ photons (Tuten et al., 2018). Because they used a $795 \mathrm{~nm}$ wavelength background, their increment thresholds are more elevated. Based on Lamb's equation (Lamb, 1995) in combination with the cone's specific spectral absorbance (Schnapf et al., 1987) the sensitivity difference between $840 \mathrm{~nm}$ and 795 $\mathrm{nm}$ is about $1.1 \log 10$ units, which is very close to the observed difference of the here reported thresholds compared to Tuten et al. (2018).

\section{The factor "Distance from PRL"}

Early studies investigating fixational eye movements reported an "optimal locus", a small group of cones that is mainly used to resolve any object of interest (Barlow, 1952; Cornsweet, 1956; Steinman, 1965). It was assumed that this retinal location colocalizes with the location of peak cone density. Scanning laser ophthalmoscopy enabled a direct study of the retinal locus used, for example during reading (Mainster et al., 1982). In combination with adaptive optics, it was observed that this optimal locus - now termed "preferred retinal locus", PRL - was displaced from the location of peak cone density (Putnam et al., 2005). Continuing investigation of the 
PRL showed that it furthermore did not colocalize with the bottom of the foveal pit or the center of the foveal avascular zone (Bedell, 1980; Zeffren et al., 1990; Wilk et al., 2017b).

In clinical research, the term PRL refers to a newly formed stable location on the retina used for fixation (Crossland, 2011), due to a central scotoma (von Noorden and Mackensen, 1962; Timberlake et al., 1986, 1987; Whittaker et al., 1988) caused by macular diseases (Crossland et al., 2005), such as age-related macular degeneration (Rees et al., 2005). As well as for healthy eyes, the underlying processes of PRL formation are yet unknown and the newly formed PRL is usually a suboptimal retinal location, given the fact that other intact locations of the retina would have provided better acuity (Bernard and Chung, 2018) or higher contrast sensitivity (Rees et al., 2005).

One of the hypotheses we assessed here, was that visual sensitivity contributes to the formation of the PRL. The overall observed fixation stability, given by the BCEA, ranged between 16 $\operatorname{arcmin}^{2}$ and $67 \operatorname{arcmin}^{2}$ in our study. This is smaller than previously reported BCEA values between $110 \operatorname{arcmin}^{2}$ and $630 \operatorname{arcmin}^{2}$ measured with SLO (Crossland and Rubin, 2002), presumably due to the increased measurement accuracy with an AOSLO. We found that, in all eyes, the location of the individual best sensitivity, as well as the empirically determined SC was offset from the PRL (Fig. 3 and 5). However, the distance between the PRL and the SC was small, about 7 arcmin on average, and given the observation that thresholds at the PRL and the 6 arcmin eccentricity were similar, we would agree with the conclusion provided by Putnam et al. 2005, that during natural viewing and the typical blur induced by the eye's optics, the visual system is insensitive to such subtle offsets, leading to a PRL formation as close as possible to the optimal location.

\section{The factor "Cone density"}

We found a moderate correlation between sensitivity thresholds and cone densities at the tested retinal locations within the fovea. Recent research on light propagation in cone photoreceptors 
would predict a similar outcome, but more clinically oriented small spot sensitivity testing comes to different conclusions. Modelling light propagation in cone inner and outer segments revealed that a smaller cone aperture is beneficial for increased quantum catch due to the cone's waveguiding properties (Meadway and Sincich, 2018). Thus, if everything else was held equal, a more densely packed cone mosaic would result in smaller foveal cones and therefore increased sensitivity. In our experiments, the diffraction limited stimulus diameter $(0.8$ arcmin FWHM) was larger than the individual foveal cone aperture (about 0.5 arcmin), favoring additional summation effects between cones straddling the incident light patch. In general, spatial summation is closely related to the field size of parasol ganglion cells (Volbrecht et al., 2000). Only within the foveola, spatial summation was found to be smaller than the field size of parasol ganglion cells, but still larger than the field size of midget ganglion cells, with a diameter of Ricco's area of 2.5 arcmin (Tuten et al., 2018). While the private line forming midget ganglion cells (Polyak, 1941; Boycott and Dowling, 1969) show a relatively stable dendritic field size up to an eccentricity of 4 degrees (Dacey, 1993), the dendritic field size of parasol ganglion cells enlarges rapidly, especially within the 5 degree eccentricity diameter (Dacey and Petersen, 1992). Because the detection of small dim stimuli seemed to rely on parasol ganglion cells, showing a steep increase of summation based on their dendritic field size and the cone's inner diameter (Curcio et al., 1990; Scoles et al., 2014), and the improved light absorption of smaller cones, a sharp peak of sensitivity within the foveola, centered on the cone density distribution should be expected. Early light sensitivity testing in a perimetry apparatus revealed such a light sensitivity peak for cone vision at the foveola (Sloan, 1939, 1950), even with a coarse testing method, with no tracking of the retinal locus and a stimulus about 1 degree in diameter. Using a smaller stimulus (10 arcmin) and testing with 0.25 degree spacing between test sites, Stiles observed a more distinct sensitivity peak within the central 1 degree radius with a slope of $2.5 \mathrm{~dB} /$ degree, surrounded by a plateau (Stiles, 1949). Further testing with clinical perimetry or fundus-controlled perimetry (so-called microperimetry) devices 
showed that the foveal sensitivity peak sharpens when stimulus size is further reduced to 6 arcmin (Goldman I size), demonstrating that sensitivity testing is prone to summation (Johnson et al., 1981; Khuu and Kalloniatis, 2015; Choi et al., 2016). Clinicians typically use a Goldmann III stimulus (24 arcmin diameter), which activates about 2500 central cones and was shown to be unable to pick up the steep foveolar sensitivity peak (Choi et al., 2016). However, recent microperimetry studies using a Goldman I stimulus reported a slope of about $1.4 \mathrm{~dB} /$ degree (Tuten et al., 2012; Khuu and Kalloniatis, 2015; Choi et al., 2016). In our study, we used a stimulus much smaller than Ricco's area of the foveola. We thus should expect an even steeper slope due to the rapid increase of cone and parasol field size. Indeed, we observed a 5 times steeper slope of about $7 \mathrm{~dB} /$ degree within the central 0.4 degree, but with a sensitivity plateau for the central 0.1 degree radius.

An ISETBio model simulating cone activation with different cone spacings was applied to assess the effect of spatial summation. This model predicted a small influence of spacing on sensitivity thresholds due to the size of our stimulus, but did not include any effects due to different light propagation in differently sized cones as proposed by Meadway and Sincich (2018) or increased summation due to an increased ganglion field size.

542 Furthermore, we found a correlation for the median foveal sensitivity threshold and the 543 participant's peak cone density. Using a clinical Humphrey field perimeter to assess sensitivity lacking fundus controlled delivery, no correlation between foveolar sensitivity and the minimum cone spacing and therefore maximum cone density with sensitivity in healthy participants was

546 found (Foote et al., 2018; Bensinger et al., 2019). However, assessing sensitivity with a

547 commercial microperimetry system, a significant correlation of sensitivity and density was

548 observed across the central \pm 5 degree eccentricity (Agarwal et al., 2015; Supriya et al., 2015;

549 Foote et al., 2019). 


\section{The factor "Outer segment length"}

Another factor influencing light sensitivity is the cone OS. Because the photopigment is stored in the OS, its length is directly linked with optical density (Bowmaker et al., 1978; Baylor et al., 1979), and therefore associated with the isomerizations and biochemical transduction cascade.

The estimation of the foveal cone's OS length from a clinical grade OCT device is still part of an ongoing discussion. At first it was reported, that the functional OS is to be found in between the 2nd and 3rd band, since the 2nd band was not the IS/OS junction but the ellipsoid zone of the IS (see also (Lu et al., 2012)) and the 3rd band was not the OS tip, but the contact cylinder (Spaide and Curcio, 2011).

Contradicting these findings it was demonstrated with an AO-OCT that the origin of these two bands are more likely to be the IS/OS junction and OS tips (Jonnal et al., 2014, 2017). Their theory is supported by a computational model simulating the light reflectivity of cones (Meadway and Sincich, 2019). But, there are still uncertainties (see Jonnal et al., 2015; Spaide, 2015), since band 2 and band 3 look very different in AO-OCT and conventional OCT. It was later stated that the thickness of band 3 may be overestimated in a conventional OCT resulting in an overestimation of OS length (Jonnal et al., 2017). Recent findings (Cuenca et al., 2018, 2020; Xie et al., 2018) support the initial report that in conventional OCT band 2 origins from the IS ellipsoids and band 3 from the phagosome zone. That is why we here decided to follow the findings reported in Spaide and Curcio (2011).

Applying these approaches to our OS length estimation we found maximum OS lengths between 28 and $40 \mu \mathrm{m}$, which is much smaller compared to other OS length reports for healthy participants from conventional OCT $(\sim 41 \mu \mathrm{m}(\mathrm{N}=43)$ (Srinivasan et al., 2008); $~ 47 \mu \mathrm{m}(\mathrm{N}=$ 23) (Wilk et al., 2017b); $52 \mu \mathrm{m}(\mathrm{N}=97$ ) (Maden et al., 2017); average values), but closer to

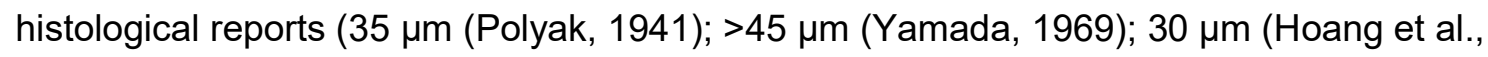
2002)). 
The shortening of the cone OS is accompanied with a decrease of photopigment density over eccentricity (Elsner et al., 1993). In accordance we found that thresholds were well correlated with foveal cone OS length, which was recently observed by Foote et al. (2019), too, but not by Bensinger et al. (2019). Studies examining this relationship of OS length and sensitivity in diseased eyes (Age-related Macular degeneration (Acton et al., 2012; Wu et al., 2014); Glaucoma (Asaoka et al., 2017); retinitis pigmentosa (but IS/OS length) (Mitamura et al., 2009), reported a significant correlation, too.

The ISETBio model confirmed this relationship, based on the assumption that the amount of photopigment per cone is relatively constant (Marcos et al., 1997). Thus, with increasing cone diameter and decreasing outer segment length over eccentricity the resulting optical density decreases.

For the four participants tested herein, we furthermore observed a direct coupling of maximum OS length and the average or peak cone density. Recent publications presented varying results about a correlation of foveal cone density and OS length: While some publications reported a significant correlation for healthy participants (Wilk et al., 2017b; Bensinger et al., 2019; Foote et al., 2019), others found no correlation between maximum cone density and OS lengths (Foote et al., 2018; Allphin et al., 2020).

\section{Variability of threshold estimates}

Taking all these observations together, we developed a model which predicted the shift of a threshold from the average threshold based on the test site's spacing, OS length and distance from the SC. This model confirmed that all these three factors are important considering sensitivity within the same retina and across different participants. However, this model showed a residual variability of \pm 0.2 log photons for the expected thresholds which could also not be explained by our ISETBio model for different cone activation patterns or cone classes spectral sensitivity. Also, this variability was more than three times higher than the average variability 
601

given by the standard deviation of $0.06 \log 10$ photons we observed during repeated testing (Fig. 3C). For further investigation of this phenomenon, we analyzed replicate testings from a piloting experiment in P2 and extended stimulation of the surrounding retinal area of a cone with conspicuous high thresholds in P3 (Fig. 11). Based on the intra run variability (STD $= \pm 0.1$ $\log 10$ photons), threshold differences up to $0.2 \log 10$ photons are not significant. Here, we observed a maximum threshold difference of $0.4 \log 10$ photons between test sites spaced only 1 arcmin apart. These additional data points reveal a very strong dependency between retinal location and small spot sensitivity, presumably caused by different weighting of individual cones. Furthermore, the replicate testing in P2 from another day confirmed that these fluctuations in sensitivity are not only on a small local scale, but also stable for a few days and can be assessed repeatable via AOSLO microstimulation.

This finding of distinct threshold variations between neighboring test sites is consistent with recent work by (Sincich et al., 2016): Using a cone sized stimulus, thresholds changed up to $100 \%$ between neighboring cones (about $0.3 \log$ units). The author's conclusion was that such unexpected observations might be caused by the individual weighting of each cone.

One possible source of variability could be minimal variations of the photopigment in the individual cones from the same retina (Schnapf et al., 1987, 1990). But this alone cannot explain such high differences between neighboring cones. A more likely explanation could be given by the observation that the individual cone input to ganglion cells varies, due to functional weighting (Chichilnisky and Baylor, 1999; Field et al., 2010; Li et al., 2014). Such functional weighting could happen at the cone-bipolar- or bipolar-ganglion-synapse. So far, in vitro studies reported linear and non-linear interaction between cones due to subunits within receptive fields of a ganglion cell (Freeman et al., 2015). Such subunits in the ganglion cell's field were reported to align directly with the bipolar cells (Liu et al., 2017).

A recent study using AOSLO-based microstimulation found that the actual test site's cone class composition has a significant influence on sensitivity thresholds (Tuten et al., 2017). The 
authors reported threshold changes of up to $100 \%$ between test sites with the same cone class composition and the target cone in a surround of a different cone class. They ruled out a simple coupling mechanism through gap junctions (Hsu et al., 2000) and propose a key role of $\mathrm{H} 1$ and $\mathrm{H} 2$ horizontal cells, mediating an elevated activation level of L-cones due to the $840 \mathrm{~nm}$ imaging background thereby inhibiting weak M-cone responses to the stimulus connected to the same horizontal cell (Thoreson and Mangel, 2012). Furthermore, they showed that neighboring Scones had a suppressing effect on thresholds. The influence of S-cones on this retinal level of modulation during our experiment in the central fovea has to be considered for the following reasons: firstly, histology showed that scattered S-cones can be found within the central 0.15 degrees (Curcio et al., 1991; Bumsted and Hendrickson, 1999), and for individual cases even within the foveola (Ahnelt, 1998). Secondly, our pulsed light source (pulse durations of about 25 psec and a frequency of $100 \mathrm{Mhz}$ ) could give rise to activate S-cones via a two photon effect (Palczewska et al., 2014). In the unlikely event of a stimulus being placed centered on an Scone, the expected threshold would have been elevated by $0.3 \log 10$ photons, based on our stimulation simulation shown in Fig. 10A. Therefore, the conspicuously high threshold found in P3 could be due to an S-cone situated at that location. processing in the lateral geniculate nucleus (Jiang et al., 2015; Alitto et al., 2019) or at later cortical stages such as V1 (Gandhi et al., 1999; Smith et al., 2006). The observed high variability between individual test sites might be also an explanation for the reported increase of rerun variability for smaller stimuli (Goldmann V versus Goldmann I) during perimetry, lacking the possibility to stabilize the stimulus on a certain retinal location (Gilpin et al., 1990; Vislisel et al., 2011). 
652

653

654

655

656

657

658

659

660

661

662

663

664

665

666

667

668

669

670

671

\section{Estimation of the number of isomerizations at threshold}

If we assumed an overall transmission of $41 \%$ for $543 \mathrm{~nm}$ light through the ocular media (Boettner and Wolter, 1962), the average threshold would be $2.9 \log 10$ photons at inner segments. Our two-dimensional model of cone capture suggested that the central cones at the target site would have absorbed between 55 photons ( $7 \%$ of the stimulus light on the retina) and 95 photons (12\%). With an optical density of about 0.5 (Bowmaker and Dartnall, 1980) this corresponds to 27 or 45 isomerizations in either L- or M-cones. The 840 imaging light had a radiant power of about $14.8 \log 10$ photons per second at the cornea $(170 \mu \mathrm{W})$. With an overall ocular transmission factor of 0.55 [840 nm; (Boettner and Wolter, 1962)] and a field size of 0.85 degree, the estimated photon rate at an individual inner segment was $10.7 \log 10$ photons per second. If a fraction of $33 \%$ was transferred into the outer segments, due to a gaussian absorption model (Macleod et al., 1992) and assuming a cone integration time (cit) of $100 \mathrm{msec}$ (Sperling and Jolliffe, 1965; Krauskopf and Mollon, 1971), we yield roughly $250 \mathrm{R}^{\star} /$ cit per Lcone and $22 \mathrm{R}^{\star} /$ cit per M-cone. Therefore, increment thresholds found here followed Weber's law (Reeves et al., 1998) comparing the L-cones isomerizations, but not for M-cones. In other words, we would have expected thresholds to vary by about 1 log unit comparing the isomerizations due to the background, but this was not the case. An explanation could be that even in the central fovea, sensitivity is not transmitted in a 1:1 circuitry but spatially summed across several cones and processed by parasol ganglion cells of the magnocellular pathway (Tuten et al., 2018). 


\section{References}

Acton JH, Theodore Smith R, Hood DC, Greenstein VC (2012) Relationship between retinal layer thickness and the visual field in early age-related macular degeneration. Investig Ophthalmol Vis Sci 53:7618-7624.

Agarwal A, Soliman MK, Hanout M, Sadiq MA, Sarwar S, Jack LS, Do D V., Nguyen QD, Sepah YJ (2015) Adaptive Optics Imaging of Retinal Photoreceptors Overlying Lesions in White Dot Syndrome and its Functional Correlation. Am J Ophthalmol 160:806-816.e2.

Ahnelt PK (1998) The photoreceptor mosaic. Eye 12:531-540.

Alitto HJ, Rathbun DL, Fisher TG, Alexander PC, Usrey WM (2019) Contrast gain control and retinogeniculate communication. Eur J Neurosci 49:1061-1068.

Allphin M, Cava J, Cooper RF, Carroll J (2020) Reevaluating Outer Segment Length as a Surrogate for Peak Cone Density. Invest Ophthalmol Vis Sci 61:204.

Arathorn DW, Yang Q, Vogel CR, Zhang Y, Tiruveedhula P, Roorda A (2007) Retinally stabilized cone-targeted stimulus delivery. Opt Express 15:13731-13744.

Asaoka R, Murata H, Yanagisawa M, Fujino $\mathrm{Y}$, Matsuura M, Inoue T, Inoue K, Yamagami J (2017) The association between photoreceptor layer thickness measured by optical coherence tomography and visual sensitivity in glaucomatous eyes. PLoS One 12:1-12.

Atchison DA, Smith G (2005) Chromatic dispersions of the ocular media of human eyes. $\mathrm{J}$ Opt Soc Am A Opt Image Sci Vis 22:29-37.

Barlow HB (1952) Eye movements during fixation. J Physiol 116:290-306.

Baylor DA, Lamb TD, Yau KW (1979) The membrane current of single rod outer segments. J Physiol 288:589-611.

Bedell HE (1980) A functional test of foveal fixation based upon differential cone directional sensitivity. Vision Res 20:557-560.

Bensinger E, Rinella N, Saud A, Loumou P, Ratnam K, Griffin S, Qin J, Porco TC, Roorda A, Duncan JL (2019) Loss of Foveal Cone Structure Precedes Loss of Visual Acuity in Patients With Rod-Cone Degeneration. Invest Ophthalmol Vis Sci 60:3187-3196.

Bernard J-BB, Chung STLL (2018) Visual Acuity Is Not the Best at the Preferred Retinal Locus in People with Macular Disease. Optom Vis Sci 95:829-836.

Boettner EA, Wolter JR (1962) Transmission of the Ocular Media. Invest Ophthalmol Vis Sci 1:776-783.

Bowmaker JK, Dartnall HJ (1980) Visual pigments of rods and cones in a human retina. J Physiol 298:501-511.

Bowmaker JK, Dartnall HJ, Lythgoe JN, Mollon JD (1978) The visual pigments of rods 
and cones in the rhesus monkey, Macaca mulatta. J Physiol 274:329-348.

Boycott BB, Dowling JE (1969) Organization of the primate retina: Light microscopy, with an appendix: A second type of midget bipolar cell in the primate retina. Philos Trans R Soc Lond B Biol Sci 255:109-184.

Bumsted K, Hendrickson A (1999) Distribution and development of short-wavelength cones differ between Macaca monkey and human fovea. J Comp Neurol 403:502516.

Chichilnisky EJ, Baylor DA (1999) Receptive-field microstructure of blue-yellow ganglion cells in primate retina. Nat Neurosci 2:889-893.

Choi AYJ, Nivison-Smith L, Khuu SK, Kalloniatis M (2016) Determining Spatial Summation and Its Effect on Contrast Sensitivity across the Central 20 Degrees of Visual Field. PLoS One 11:e0158263.

Cornsweet TN (1956) Determination of the Stimuli for Involuntary Drifts and Saccadic Eye Movements. J Opt Soc Am 46:987.

Cottaris NP, Jiang H, Ding X, Wandell BA, Brainard DH (2019) A computationalobserver model of spatial contrast sensitivity: Effects of wave-front-based optics, cone-mosaic structure, and inference engine. J Vis 19:8.

Cottaris NP, Wandell BA, Rieke F, Brainard DH (2020) A computational observer model of spatial contrast sensitivity: Effects of photocurrent encoding, fixational eye movements, and inference engine. J Vis 20:17.

Crossland MD (2011) THE PREFERRED RETINAL LOCUS IN Toward A Consensus Definition. J Retin Vitr Dis:2109-2114.

Crossland MD, Culham LE, Kabanarou SA, Rubin GS (2005) Preferred retinal locus development in patients with macular disease. Ophthalmology 112:1579-1585.

Crossland MD, Rubin GS (2002) The use of an infrared eyetracker to measure fixation stability. Optom Vis Sci 79:735-739.

Cuenca N, Ortuño-Lizarán I, Pinilla I (2018) Cellular Characterization of OCT and Outer Retinal Bands Using Specific Immunohistochemistry Markers and Clinical Implications. Ophthalmology 125:407-422.

Cuenca N, Ortuño-Lizarán I, Sánchez-Sáez X, Kutsyr O, Albertos-Arranz H, FernándezSánchez L, Martínez-Gil N, Noailles A, López-Garrido JA, López-Gálvez M, Lax P, Maneu V, Pinilla I (2020) Interpretation of OCT and OCTA images from a histological approach: Clinical and experimental implications. Prog Retin Eye Res 77:100828.

Cunefare D, Fang L, Cooper RF, Dubra A, Carroll J (2017) Open source software for automatic detection of cone photoreceptors in adaptive optics ophthalmoscopy using convolutional neural networks. Sci Rep:1-11.

Curcio C a, Allen K a, Sloan KR, Lerea CL, Hurley JB, Klock IB, Milam a H (1991) 
Distribution and morphology of human cone photoreceptors stained with anti-blue opsin. J Comp Neurol 312:610-624.

Curcio CA, Sloan KR, Kalina RE, Hendrickson AE (1990) Human photoreceptor topography. J Comp Neurol 292:497-523.

Dacey DM (1993) The mosaic of midget ganglion cells in the human retina. J Neurosci 13:5334-5355.

Dacey DM, Petersen MR (1992) Dendritic field size and morphology of midget and parasol ganglion cells of the human retina. Proc Natl Acad Sci U S A 89:96669670.

Domdei N, Domdei L, Reiniger JL, Linden M, Holz FG, Roorda A, Harmening WM (2018) Ultra-high contrast retinal display system for single photoreceptor psychophysics. Biomed Opt Express 9:157-172.

Domdei N, Linden M, Reiniger JL, Holz FG, Harmening WM (2019) Eye tracking-based estimation and compensation of chromatic offsets for multi-wavelength retinal microstimulation with foveal cone precision. Biomed Opt Express 10:4126-4141.

Elsner AE, Burns SA, Webb RH (1993) Mapping cone photopigment optical density. J Opt Soc Am A 10:52.

Field GD, Gauthier JL, Sher A, Greschner M, Machado TA, Jepson LH, Shlens J, Gunning DE, Mathieson K, Dabrowski W, Paninski L, Litke AM, Chichilnisky EJ (2010) Functional connectivity in the retina at the resolution of photoreceptors. Nature 467:673-677.

Foote KG, Huerta I de la, Gustafson K, Baldwin A, Zayit-Soudry S, Rinella N, Porco TC, Roorda A, Duncan JL (2019) Cone spacing correlates with retinal thickness and microperimetry in patients with inherited retinal degenerations. Investig Ophthalmol Vis Sci 60:1234-1243.

Foote KG, Loumou P, Griffin S, Qin J, Ratnam K, Porco TC, Roorda A, Duncan JL (2018) Relationship Between Foveal Cone Structure and Visual Acuity Measured With Adaptive Optics Scanning Laser Ophthalmoscopy in Retinal Degeneration. Invest Ophthalmol Vis Sci 59:3385-3393.

Freeman J, Field GD, Li PH, Greschner M, Gunning DE, Mathieson K, Sher A, Litke AM, Paninski L, Simoncelli EP, Chichilnisky EJ (2015) Mapping nonlinear receptive field structure in primate retina at single cone resolution. Elife 4:1-21.

Gandhi SP, Heeger DJ, Boynton GM (1999) Spatial attention affects brain activity in human primary visual cortex. Proc Natl Acad Sci U S A 96:3314-3319.

Geisler WS, Davila KD (1985) Ideal discriminators in spatial vision:two-point stimuli. J Opt Soc Am A 2:1483.

Gilpin LB, Stewart WC, Hunt HH, Broom CD (1990) Threshold variability using different Goldmann stimulus sizes. Acta Ophthalmol 68:674-676. 
Goldberg ME, Walker MF (2013) The control of Gaze. In: Principles of Neural Science, 5th ed. (Kandel ER, Schwartz JH, Jessell TM, Siegelbaum SA, Hudspeth AJ, eds), pp 894-916. New York: McGraw-Hill.

Grieve K, Tiruveedhula P, Zhang Y, Roorda A (2006) Multi-wavelength imaging with the adaptive optics scanning laser Ophthalmoscope. Opt Express 14:12230-12242.

Harmening WM, Tiruveedhula P, Roorda A, Sincich LC (2012) Measurement and correction of transverse chromatic offsets for multi-wavelength retinal microscopy in the living eye. Biomed Opt Express 3:2066-2077.

Harmening WM, Tuten WS, Roorda A, Sincich LC (2014) Mapping the perceptual grain of the human retina. J Neurosci 34:5667-5677.

Hoang Q V., Linsenmeier RA, Chung CK, Curcio CA (2002) Photoreceptor inner segments in monkey and human retina: Mitochondrial density, optics, and regional variation. Vis Neurosci 19:395-407.

Hsu A, Smith RG, Buchsbaum G, Sterling P (2000) Cost of cone coupling to trichromacy in primate fovea. J Opt Soc Am A Opt Image Sci Vis 17:635-640.

Jiang Y, Yampolsky D, Purushothaman G, Casagrande VA (2015) Perceptual decision related activity in the lateral geniculate nucleus. J Neurophysiol 114:717-735.

Johnson CA, Keltner JL, Balestrery FG (1981) Static and acuity profile perimetry at various adaptation levels. Doc Ophthalmol 50:371-388.

Jonnal RS, Gorczynska I, Migacz J V., Azimipour M, Zawadzki RJ, Werner JS (2017) The properties of outer retinal band three investigated with adaptive-optics optical coherence tomography. Investig Ophthalmol Vis Sci 58:4559-4568.

Jonnal RS, Kocaoglu OP, Zawadzki RJ, Lee S-H, Werner JS, Miller DT (2015) Author Response: Outer Retinal Bands. Investig Opthalmology Vis Sci 56:2507.

Jonnal RS, Kocaoglu OP, Zawadzki RJ, Lee S-HSHS-H, Werner JS, Miller DT (2014) The cellular origins of the outer retinal bands in optical coherence tomography images. Investig Ophthalmol Vis Sci 55:7904-7918.

Khuu SK, Kalloniatis M (2015) Standard automated perimetry: Determining spatial summation and its effect on contrast sensitivity across the visual field. Investig Ophthalmol Vis Sci 56:3565-3576.

King-Smith PE, Grigsby SS, Vingrys AJ, Benes SC, Supowit A (1994) Efficient and unbiased modifications of the QUEST threshold method: Theory, simulations, experimental evaluation and practical implementation. Vision Res 34:885-912.

Koenig D, Hofer H (2011) The absolute threshold of cone vision. J Vis 11:1-24.

Krauskopf J, Mollon JD (1971) The independence of the temporal integration properties of individual chromatic mechanisms in the human eye. J Physiol 219:611-623.

Lamb TD (1995) Photoreceptor spectral sensitivities: Common shape in the longwavelength region. Vision Res 35:3083-3091. 
Li PH, Field GD, Greschner M, Ahn D, Gunning DE, Mathieson K, Sher A, Litke AM, Chichilnisky EJ (2014) Retinal Representation of the Elementary Visual Signal. Neuron 81:130-139.

Liu JK, Schreyer HM, Onken A, Rozenblit F, Khani MH, Krishnamoorthy V, Panzeri S, Gollisch T (2017) Inference of neuronal functional circuitry with spike-triggered nonnegative matrix factorization. Nat Commun 8.

Lu R-W, Curcio CA, Zhang Y, Zhang Q-X, Pittler SJ, Deretic D, Yao X-C (2012) Investigation of the hyper-reflective inner/outer segment band in optical coherence tomography of living frog retina. J Biomed Opt 17:060504.

Macleod DIAA, Williams DR, Makous W (1992) A visual nonlinearity fed by single cones. Vision Res 32:347-363.

Maden G, Cakir A, Icar D, Erden B, Bolukbasi S, Elcioglu M (2017) The Distribution of the Photoreceptor Outer Segment Length in a Healthy Population. J Ophthalmol 2017.

Mainster MA, Timberlake GT, Webb RH, Hughes GW (1982) Scanning laser ophthalmoscopy. Clinical applications. Ophthalmology 89:852-857.

Marcos S, Tornow RP, Elsner AE, Navarro R (1997) Foveal cone spacing and cone photopigment density difference: Objective measurements in the same subjects. Vision Res 37:1909-1915.

Marriott FHC (1963) The foveal absolute visual threshold for short flashes and small fields. J Physiol 169:416-423.

Meadway A, Sincich LC (2018) Light propagation and capture in cone photoreceptors. Biomed Opt Express 9:5543.

Meadway A, Sincich LC (2019) Light reflectivity and interference in cone photoreceptors. Biomed Opt Express 10:6531.

Mitamura Y, Aizawa S, Baba T, Hagiwara A, Yamamoto S (2009) Correlation between retinal sensitivity and photoreceptor inner/outer segment junction in patients with retinitis pigmentosa. $\mathrm{Br} J$ Ophthalmol 93:126-127.

Nachmias J (1959) Two-dimensional motion of the retinal image during monocular fixation. J Opt Soc Am 49:901-908.

Palczewska G, Vinberg F, Stremplewski P, Bircher MP, Salom D, Komar K, Zhang J, Cascella M, Wojtkowski M, Kefalov VJ, Palczewski K (2014) Human infrared vision is triggered by two-photon chromophore isomerization. Proc Natl Acad Sci U S A 111:E5445-5454.

Poletti M, Listorti C, Rucci M (2013) Microscopic eye movements compensate for nonhomogeneous vision within the fovea. Curr Biol 23:1691-1695.

Polyak SL (1941) The Retina. Chicago: University of Chicago Press.

Poonja S, Patel S, Henry L, Roorda A (2005) Dynamic visual stimulus presentation in 
an adaptive optics scanning laser ophthalmoscope. J Refract Surg 21:S575-S580.

Pritchard RM (1961) Stabilized images on the retina. Sci Am 204:72-78.

Provis JM, Dubis AM, Maddess T, Carroll J (2013) Adaptation of the central retina for high acuity vision: Cones, the fovea and the a vascular zone. Prog Retin Eye Res 35:63-81.

Putnam NM, Hofer HJ, Doble N, Chen L, Carroll J, Williams DR (2005) The locus of fixation and the foveal cone mosaic. J Vis 5:632-639.

Rees AL, Kabanarou SA, Culham LE, Rubin G. (2005) Can retinal eccentricity predict visual acuity and contrast sensitivity at the PRL in AMD patients? Int Congr Ser 1282:694-698.

Reeves A, Wu S, Schirillo J (1998) The effect of photon noise on the detection of white flashes. Vision Res 38:691-703.

Reiniger JL, Lobecke AC, Sabesan R, Bach M, Verbakel F, de Brabander J, Holz FG, Berendschot TTJM, Harmening WM (2019) Habitual higher order aberrations affect Landolt but not Vernier acuity. J Vis.

Sakitt B (1972) Counting every quantum. J Physiol 223:131-150.

Schnapf JL, Kraft TW, Baylor D a. (1987) Spectral sensitivity of human cone photoreceptors. Nature 325:439-441.

Schnapf JL, Nunn BJ, Meister M, Baylor DA (1990) Visual transduction in cones of the monkey Macaca fascicularis. J Physiol 427:681-713.

Scoles D, Sulai YN, Langlo CS, Fishman GA, Curcio CA, Carroll J, Dubra A (2014) In vivo imaging of human cone photoreceptor inner segments. Invest Ophthalmol Vis Sci 55:4244-4251.

Sincich LC, Sabesan R, Tuten WS, Roorda A, Harmening WM (2016) Functional Imaging of Cone Photoreceptors. In: Human Color Vision (Kremers J, Baraas RC, Marshall NJ, eds), pp 71-104. Cham: Springer International Publishing.

Sloan LL (1939) INSTRUMENTS AND TECHNICS FOR THE CLINICAL TESTING OF LIGHT SENSE. Arch Ophthalmol 21:913-934.

Sloan LL (1950) The threshold gradients of the rods and the cones: In the dark-adapted and in the partially light-adapted eye. Am J Ophthalmol 33:1077-1089.

Slonaker JR (1897) A comparative study of the area of acute vision in vertebrates. J Morphol 13:445-502.

Smith AT, Cotillon-Williams NM, Williams AL (2006) Attentional modulation in the human visual cortex: The time-course of the BOLD response and its implications. Neuroimage 29:328-334.

Spaide RF (2015) Outer retinal bands. Investig Ophthalmol Vis Sci 56:2505-2506.

Spaide RF, Curcio CA (2011) Anatomical correlates to the bands seen in the outer 
retina by optical coherence tomography: Literature review and model. Retina 31:1609-1619.

Sperling HG, Jolliffe CL (1965) Intensity-Time Relationship at Threshold for Spectral Stimuli in Human Vision*. J Opt Soc Am 55:191.

Srinivasan VJ, Monson BK, Wojtkowski M, Bilonick RA, Gorczynska I, Chen R, Duker JS, Schuman JS, Fujimoto JG (2008) Characterization of outer retinal morphology with high-speed, ultrahigh-resolution optical coherence tomography. Investig Ophthalmol Vis Sci 49:1571-1579.

Steinman RM (1965) Effect of Target Size, Luminance, and Color on Monocular Fixation. J Opt Soc Am 55:1158.

Stiles WS (1949) Increment thresholds \&amp; the mechanisms of colour vision. Doc Ophthalmol 3:138-165.

Supriya D, Shwetha M, Kiran Anupama K, Kummelil Mathew K, Berendschot TTJM, Schouten JSAG, Bharamshetter R, Naresh YK, Rohit S, Hegde B (2015) Structural and function correlation of cone packing utilizing adaptive optics and microperimetry. Biomed Res Int 2015.

Takeshita D, Smeds L, Ala-Laurila P (2017) Processing of single-photon responses in the mammalian On and Off retinal pathways at the sensitivity limit of vision. Philos Trans R Soc Lond B Biol Sci 372.

Thoreson WB, Mangel SC (2012) Lateral interactions in the outer retina. Prog Retin Eye Res 31:407-441.

Timberlake GT, Mainster MA, Peli E, Augliere RA, Essock EA, Arend LE (1986) Reading with a macular scotoma. I. Retinal location of scotoma and fixation area. Investig Ophthalmol Vis Sci 27:1137-1147.

Timberlake GT, Peli E, Essock EA, Augliere RA (1987) Reading with a macular scotoma. II. Retinal locus for scanning text. Investig Ophthalmol Vis Sci 28:12681274.

Tinsley JN, Molodtsov MI, Prevedel R, Wartmann D, Espigulé-Pons J, Lauwers M, Vaziri A (2016) Direct detection of a single photon by humans. Nat Commun $7: 12172$.

Tuten WS, Cooper RF, Tiruveedhula P, Dubra A, Roorda A, Cottaris NP, Brainard DH, Morgan JIWW (2018) Spatial summation in the human fovea: Do normal optical aberrations and fixational eye movements have an effect? J Vis 18:6.

Tuten WS, Harmening WM, Sabesan R, Roorda A, Sincich LC (2017) Spatiochromatic Interactions between Individual Cone Photoreceptors in the Human Retina. J Neurosci 37:9498-9509.

Tuten WS, Tiruveedhula P, Roorda A (2012) Adaptive optics scanning laser ophthalmoscope-based microperimetry. Optom Vis Sci 89:563-574. 
Vislisel JM, Doyle CK, Johnson CA, Wall M (2011) Variability of rarebit and standard perimetry sizes i and III in normals. Optom Vis Sci 88:635-639.

Volbrecht VJ, Shrago EE, Schefrin BE, Werner JS (2000) Spatial summation in human cone mechanisms from 0 degrees to 20 degrees in the superior retina. J Opt Soc Am A Opt Image Sci Vis 17:641-650.

von Noorden GK, Mackensen G (1962) Phenomenology of eccentric fixation. Am J Ophthalmol 53:642-661.

Wang Y, Bensaid N, Tiruveedhula P, Ma J, Ravikumar S, Roorda A (2019) Human foveal cone photoreceptor topography and its dependence on eye length. Elife 8.

Watson AB, Pelli DG (1983) QUEST: a Bayesian adaptive psychometric method. Percept Psychophys 33:113-120.

Whittaker SG, Budd J, Cummings RW (1988) Eccentric fixation with macular scotoma. Investig Ophthalmol Vis Sci 29:268-278.

Wilk MA, Dubis AM, Cooper RF, Summerfelt P, Dubra A, Carroll J (2017a) Assessing the spatial relationship between fixation and foveal specializations. Vision Res 132:53-61.

Wilk MA, Wilk BM, Langlo CS, Cooper RF, Carroll J (2017b) Evaluating outer segment length as a surrogate measure of peak foveal cone density. Vision Res 130:57-66.

Wu Z, Ayton LN, Luu CD, Guymer RH (2014) Relationship between retinal microstructures on optical coherence tomography and microperimetry in agerelated macular degeneration. Ophthalmology 121:1445-1452.

Xie W, Zhao M, Tsai SH, Burkes WL, Potts LB, Xu W, Payne HR, Hein TW, Kuo L, Rosa RH (2018) Correlation of spectral domain optical coherence tomography with histology and electron microscopy in the porcine retina. Exp Eye Res 177:181-190.

Yamada E (1969) Some structural features of the fovea centralis in the human retina. Arch Ophthalmol 82:151-159.

Yuodelis C, Hendrickson A (1986) A qualitative and quantitative analysis of the human fovea during development. Vision Res 26:847-855.

Zeffren BS, Applegate RA, Bradley A, van Heuven WA (1990) Retinal fixation point location in the foveal avascular zone. Invest Ophthalmol Vis Sci 31:2099-2105.

Zhang C, Kim YJ, Silverstein AR, Hoshino A, Reh TA, Dacey DM, Wong RO (2020) Circuit Reorganization Shapes the Developing Human Foveal Midget Connectome toward Single-Cone Resolution. Neuron 108:905-918.e3. 

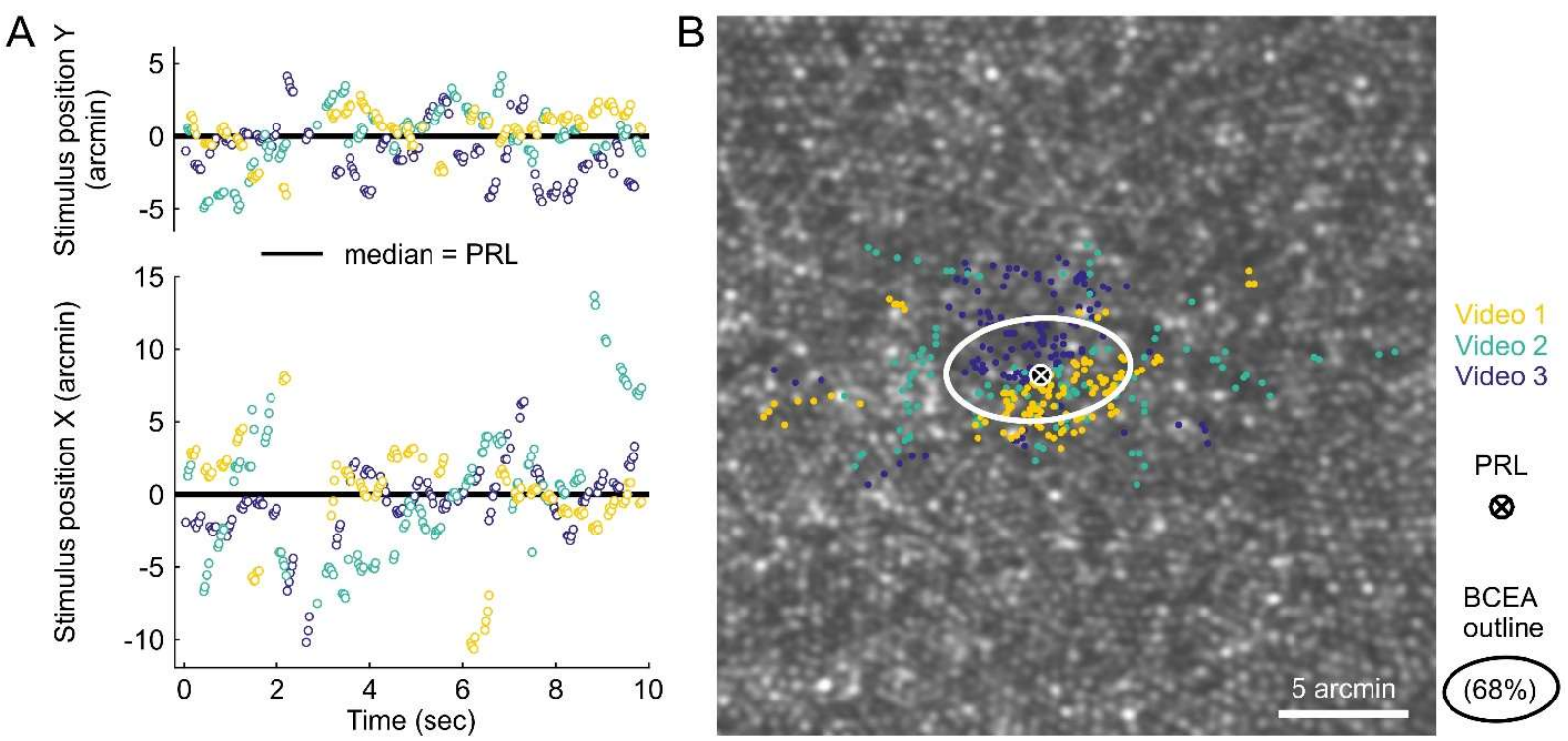

973 Figure 1: Preferred retinal locus (PRL) determination from three $10 \mathrm{sec}$ videos. A) The nominal

974 fixation target was a $1.6 \times 1.6$ arcmin square presented at a fixed position in the center of the

975 AOSLO raster flashing with $3 \mathrm{~Hz}$. High resolution eye motion traces were recorded in three 10

976 second epochs, tracking the position of the target's center in retinal coordinates. Single dots

977 represent frame-by-frame derived retinal coordinates of the target, colors indicate repeats. B)

978 Stimulus positions in relationship to the foveal cone photoreceptor mosaic. The bivariate contour 979 ellipse was set to contain $68 \%$ of all stimulus locations. The participant's PRL (white ellipse and 980 marker) was computed as the median data point, pooling all locations of the three consecutively recorded videos. 

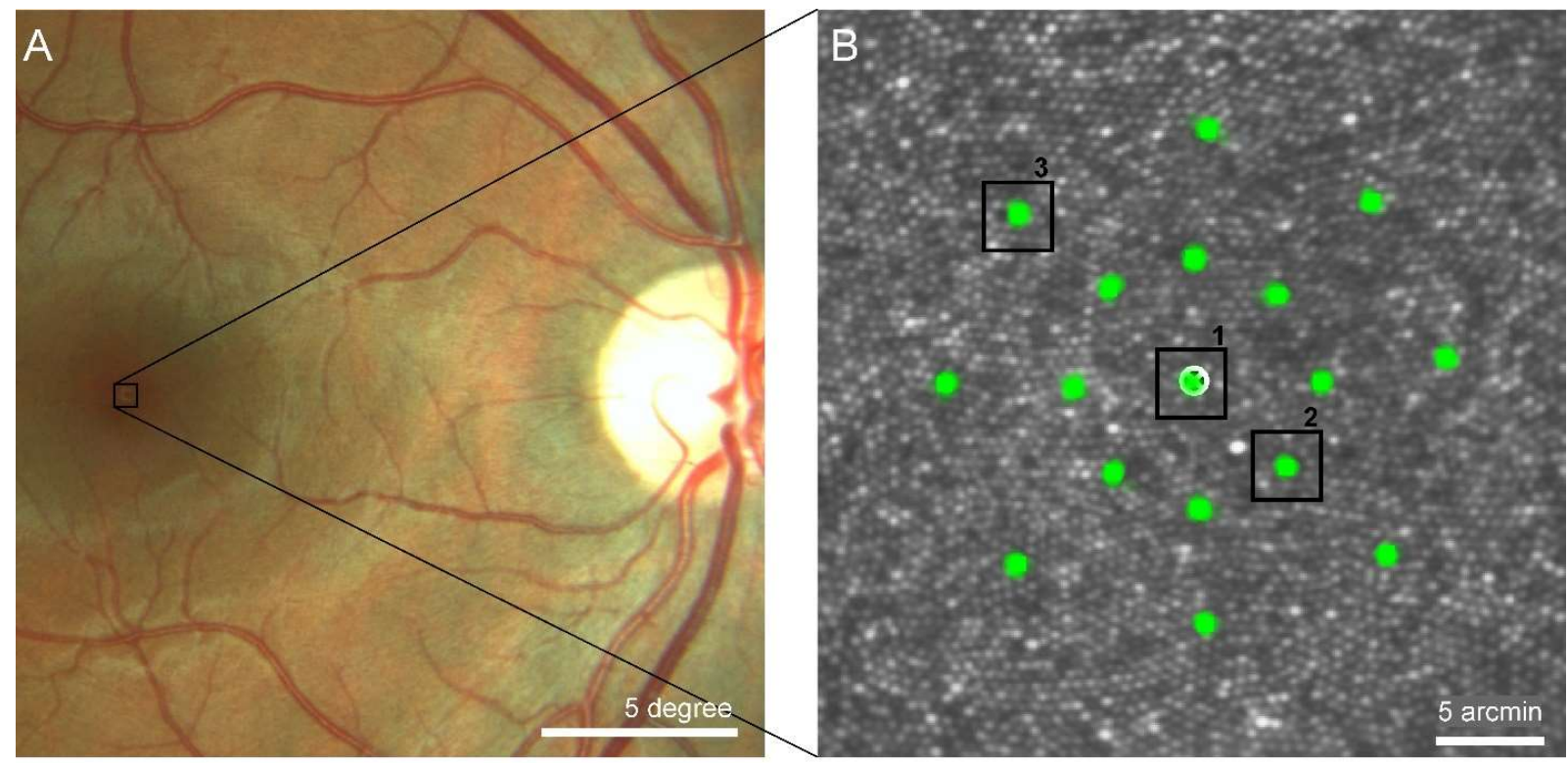
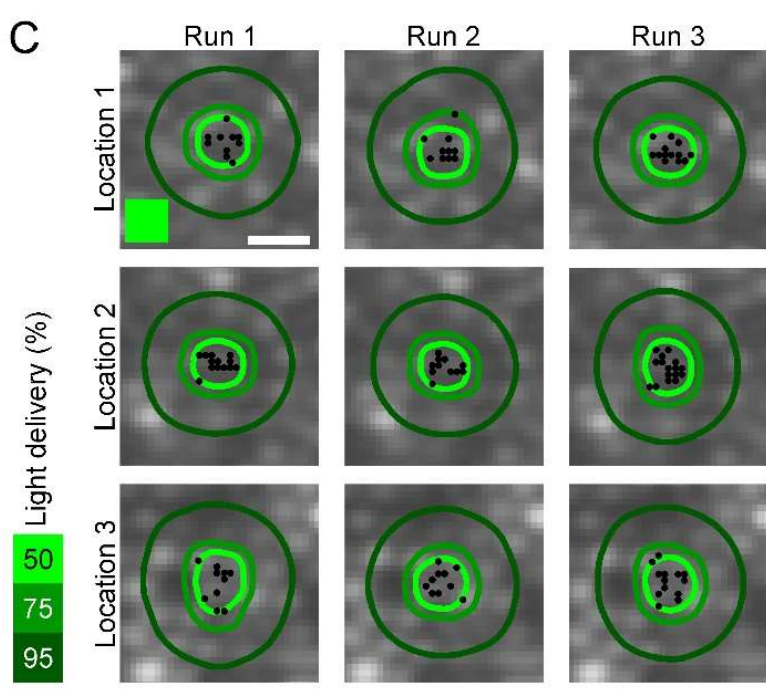
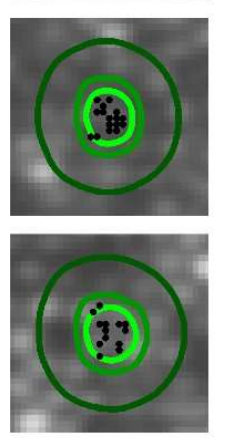

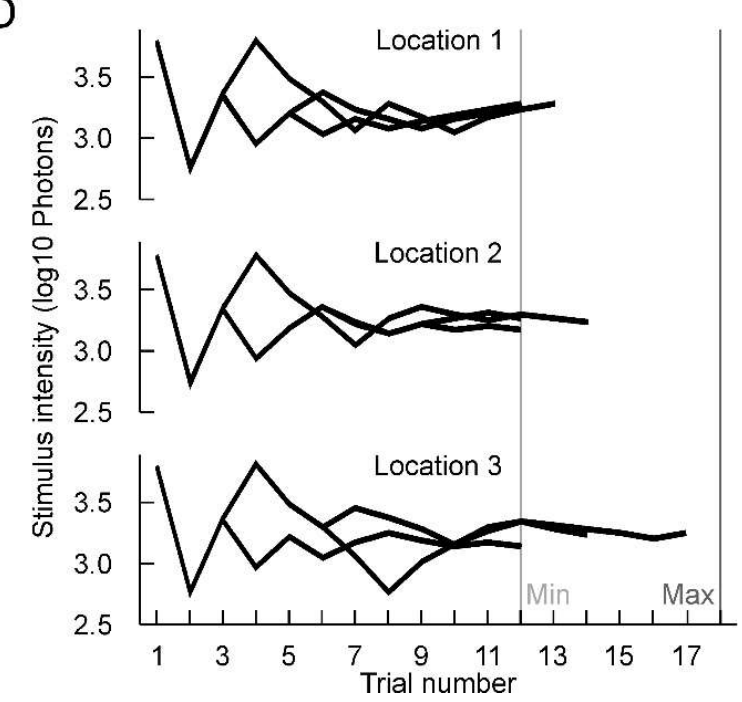

984

985

986

987

988

989

990

991

992

993

Figure 2: AOSLO-based microstimulation. A) Color fundus image of one participant. The grey square shows the size and position of the AOSLO imaging and stimulation raster on the retina, positioned at the center of the fovea. B) Cropped view of an averaged frame showing the AOSLO image of the central fovea. Retinal stimulation sites are marked by transparent green markers, three sites are highlighted by black boxes to be further analyzed in the next panels. C) Zoomed-in view of selected target sites. Markers indicate individual stimulus locations during repeated stimulation for threshold estimation. The green square represents the stimulus in raster pixel size. The contour lines mark $50 \%, 75 \%$, and $95 \%$ of the summed light delivery for each run. The scale bar is 1 arcmin. D) Exemplary progression of stimulus intensity based on the current threshold estimation via QUEST with three runs per test site. The individual run was 
A

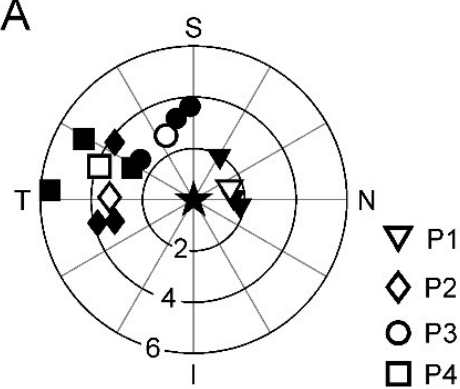

PRL position (arcmin)
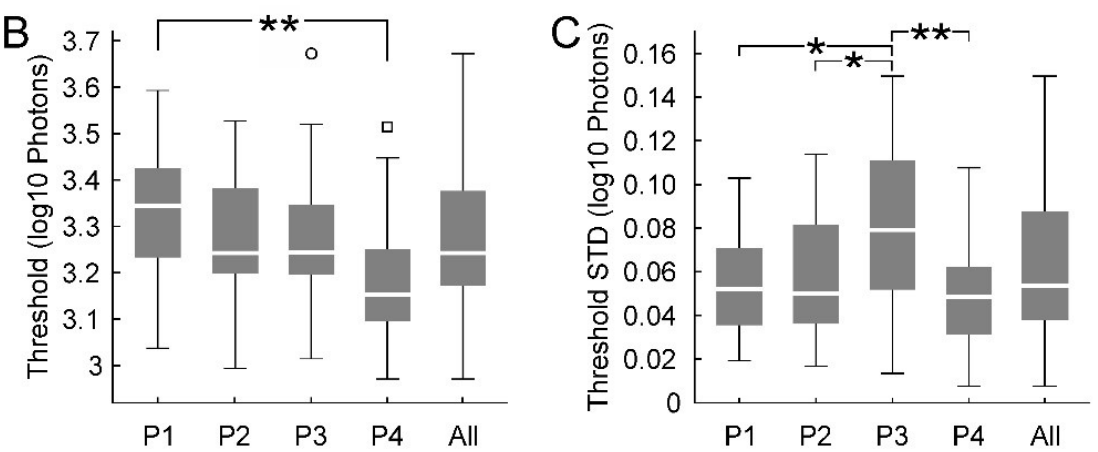

Figure 3: Fixation behavior and foveal increment sensitivity. A) The PRL of each participant (P1-

4 ) is plotted relative to the location of the cone density centroid (plot origin, 'star' marker). Filled markers represent single measurements, open markers are the final PRL based on three repeat measurements. Letters marking Nasal, Temporal, Inferior, and Superior. B) Pooled sensitivity thresholds across all test sites for each participant separately (P1-P4), and combined (“All”). While the range ( $\sim 0.5 \log 10$ units, whiskers), as well as the highest and lowest thresholds, were very similar across participants, the median, 1st, and 3rd quartile differed. C) The observed standard deviation (STD) of repeated threshold estimations at the same test site was similar for three participants. Variability for P3 was higher than for the other three. $85 \%$ of all observed STDs were less or equal to $0.10 \log 10$ photons. 

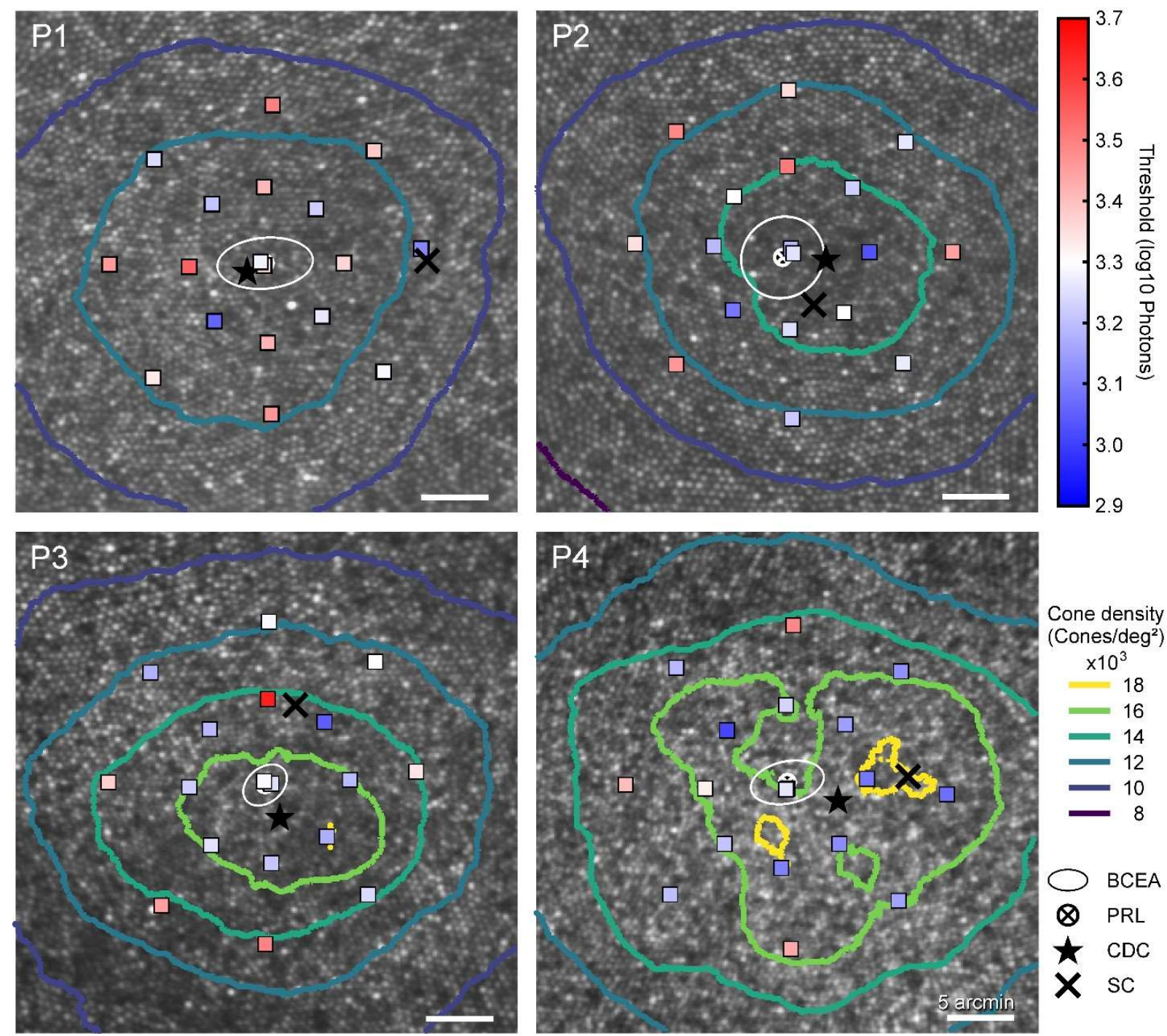

1011 Figure 4: Sensitivity in relationship to cone topography and fixation behavior in all participants.

1012 Squares mark the average stimulus location for each test site, coloring reflects the median

1013 sensitivity threshold in log10 photons. Marker area is four times the actual stimulus area on the 1014 retina and equals the average $95 \%$ outline of the summed light delivery (see Fig.1C). Cone 1015 density is indicated by colored contour lines. The PRL (white circle and crosshair), the location 1016 of the cone density centroid (star) and an estimated location of the sensitivity centroid (SC, 1017 cross) are shown as well. 

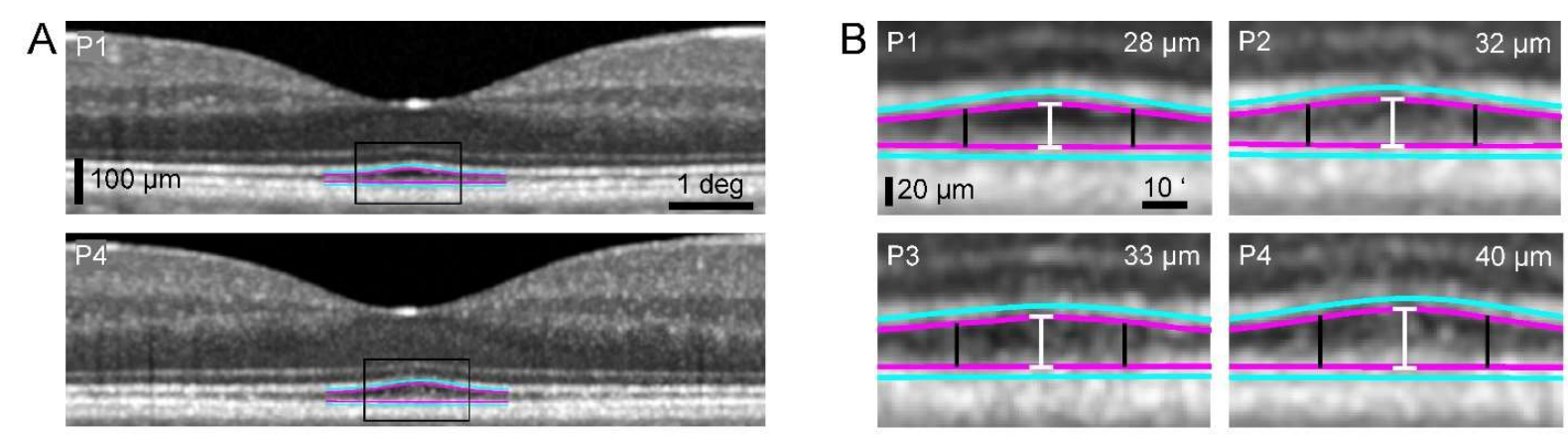

C
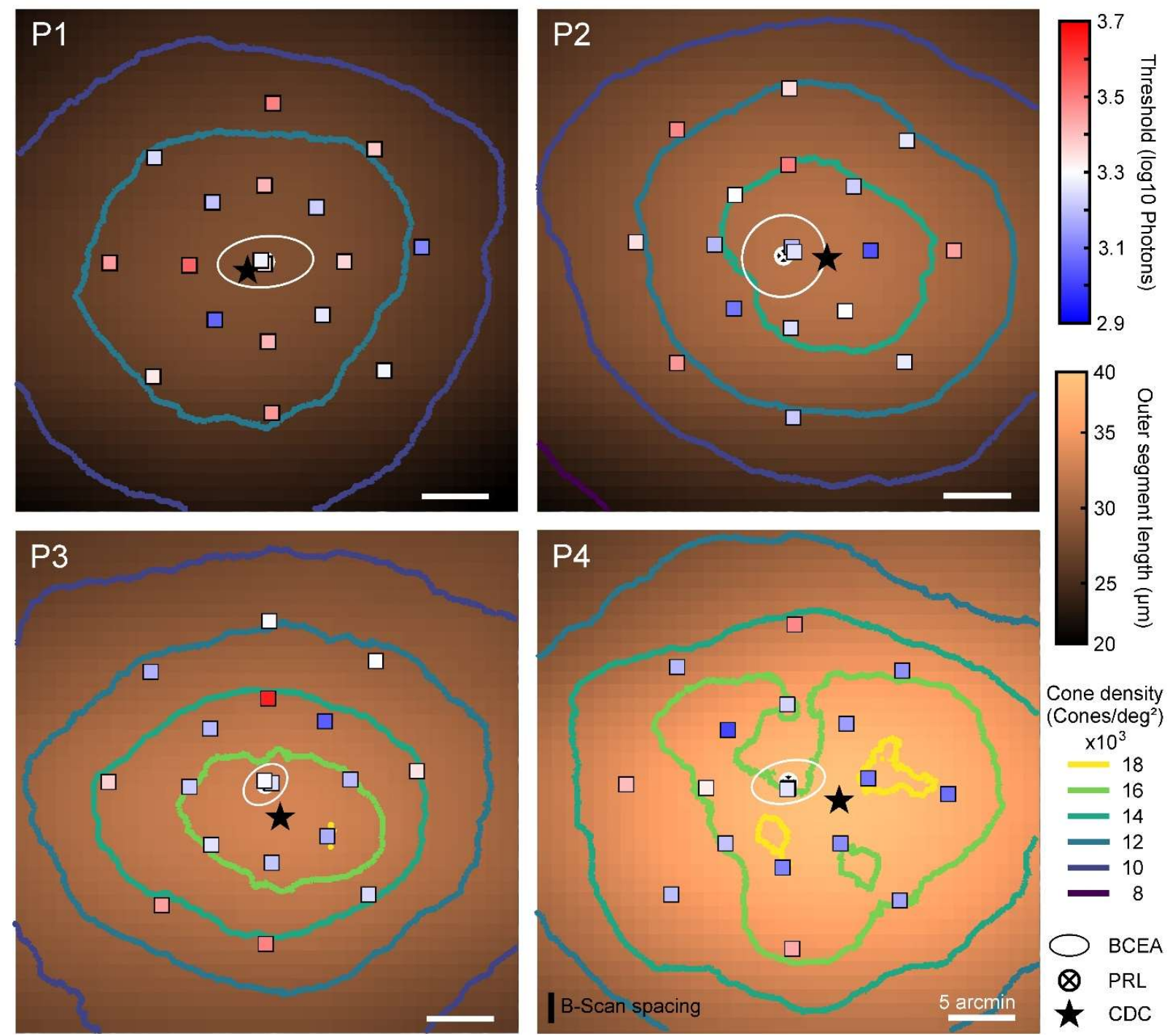

Figure 5: Cone outer segment length topography. A) Foveal OCT B-scans from P1 and P4, and a zoomed-in view of the OS in the central fovea. B) Further zoomed-in view of central OS and measurement demonstration for all 4 participants. The band's center (cyan line) was semiautomatically marked. The OS start and end (magenta) was found by fitting a 1D gaussian 

right corner. C) Interpolated 2D map of cone OS length in the central fovea for each participant. The black vertical lines in $(B)$ indicate the region of the $2 \mathrm{D}$ presentation in $(C)$. 

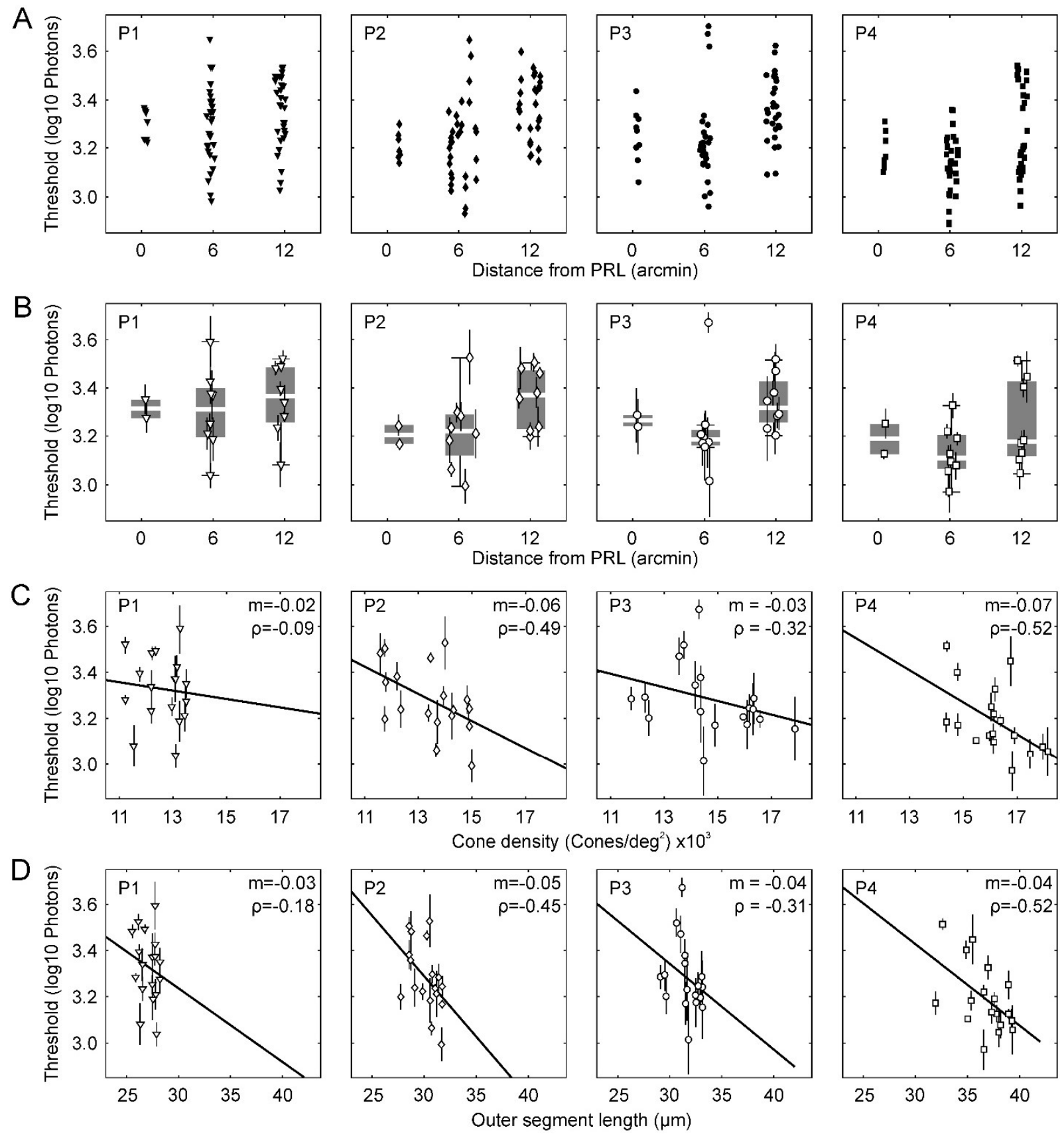

Figure 6: Sensitivity thresholds for each participant in relation to distance from PRL, cone density and outer segment length. A) Individual thresholds for each test site's distance from the PRL are marked with filled symbols. B) For each test site we used the median threshold (open symbols) of repeated testing (3-5), and reported this value as the test site's sensitivity. Boxplots show median (white line), first and third quartile (box), whiskers extend to 1.5 fold the distance between the first and third quartile. C) Median thresholds in relation to the test site's cone density. D) Median thresholds in relation to the outer segment length at the test site as obtained 
from the 2-D map shown in Fig. 5. Vertical lines in B, C, and D show the standard deviation across repeated runs, thick black lines are linear fits to the data.

A

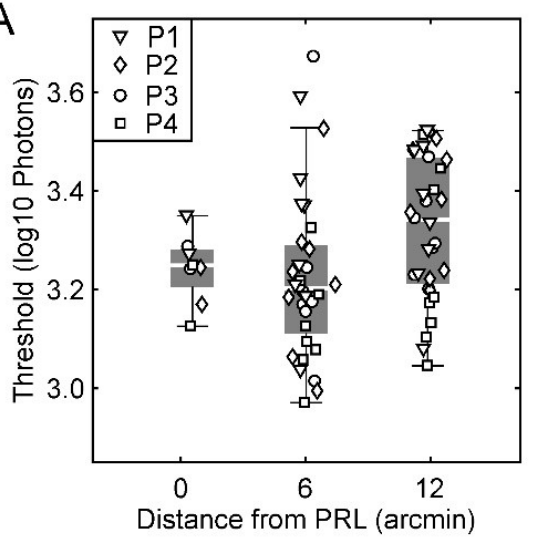

B

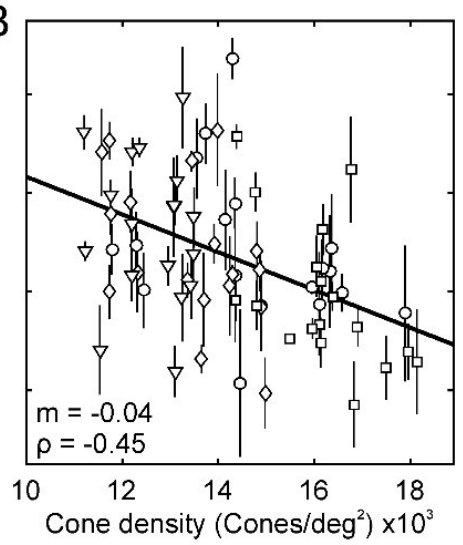

C

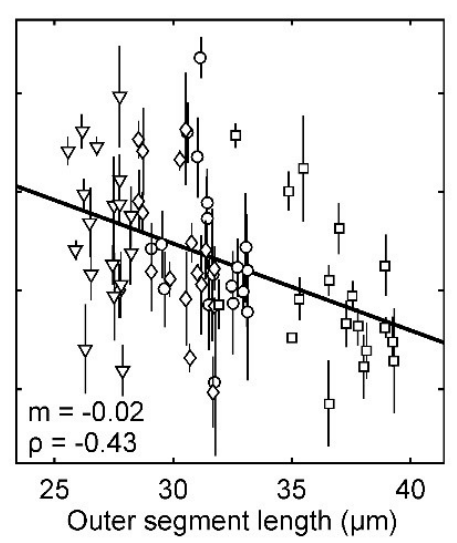

Figure 7: Pooling threshold data across subjects. A) Sensitivity thresholds of all participants as a function of test site distance from PRL, given as median (white line), first and third quartile (box). Whiskers extend to 1.5 fold the distance between the first and third quartile. For the pooled data set, a bend towards lower thresholds at 6 arcmin was observed. B) Thresholds as a function of local cone density at the test site, vertical lines show the standard deviation of threshold estimates for repeated testing. Thresholds and cone densities showed a moderate correlation ( $\rho$ $=-0.45)$. C) Thresholds as a function of cone outer segment lengths showing a moderate correlation $(\rho=-0.43$ ). All independent variables (Distance, Density, OS length) were pairwise significantly correlated with each other ( $p<0.001$, data not shown). 
A

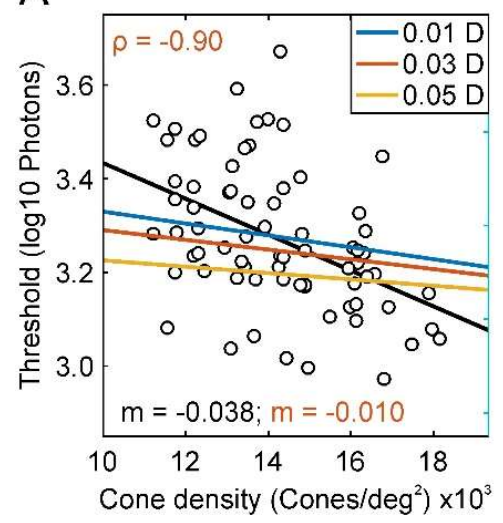

$B$

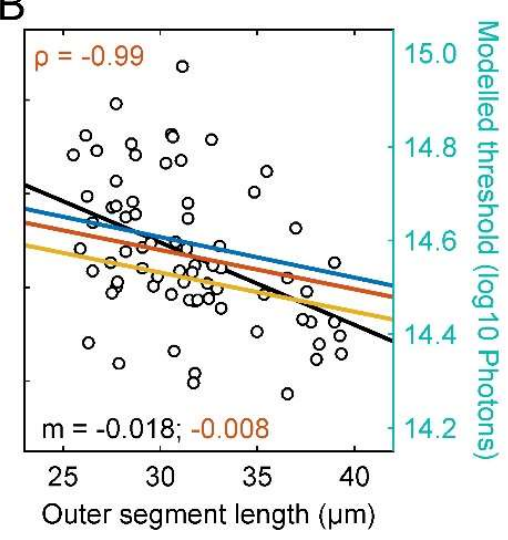

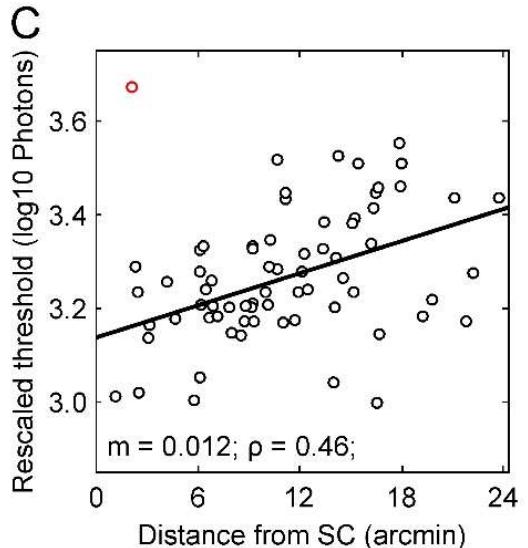

1053

1054

1055

1056

1057

1058

1059

1060

1061

1062

1063

Figure 8: ISETBio model testing the impact of cone density and outer segment (OS) length. A)

The ISETBio model evaluating the threshold photons to elicit a certain number of isomerizations, fitted for $0.01 \mathrm{D}, 0.03 \mathrm{D}$, and $0.05 \mathrm{D}$ residual defocus. The simulation revealed only a small influence of cone density and therefore spacing on sensitivity thresholds $(\mathrm{m}=$ 0.010 and $\rho=-0.90$ with $0.03 \mathrm{D}$ ), too small to explain the observations. B) To test the influence of outer segment length and therefore optical density on sensitivity, we fed the observed range of outer segment lengths $(25 \mu \mathrm{m}$ to $40 \mu \mathrm{m})$ in our ISETBio model, which predicted a strong impact of different outer segment lengths on thresholds $(m=-0.008$ and $\rho=-0.99$ with $0.03 D)$. C) The pooled data of the rescaled thresholds, which had the OS length and density influence removed (see methods), against the distance from the participant's sensitivity centroid (see Fig. 4). The red marked threshold was ignored in this analysis due to its uncertain nature. 


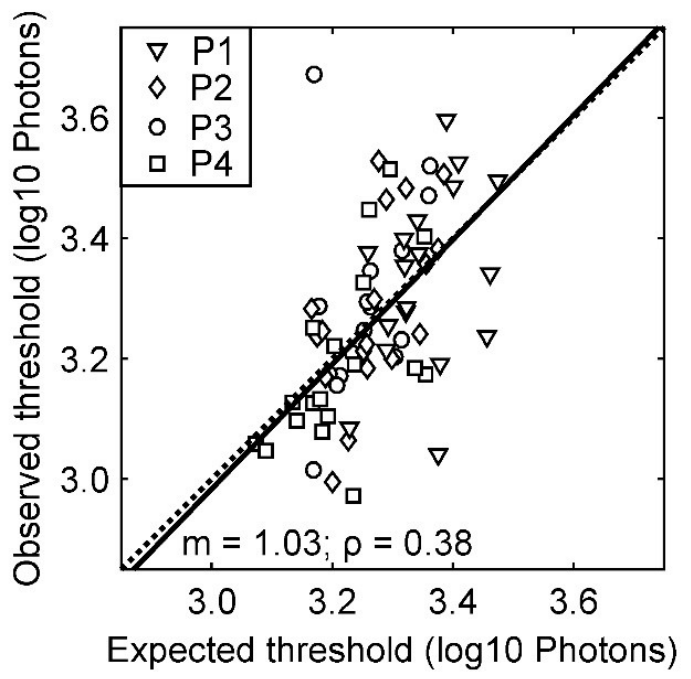

Figure 9: Predicting sensitivity thresholds from retinal factors. The expected threshold was derived from the test site's cone density, OS length and distance from the sensitivity centroid (Fig. 4). Each parameter was converted into a threshold offset from the average. The solid line is the linear fit to the data, the dotted line shows the 1:1 relationship. 

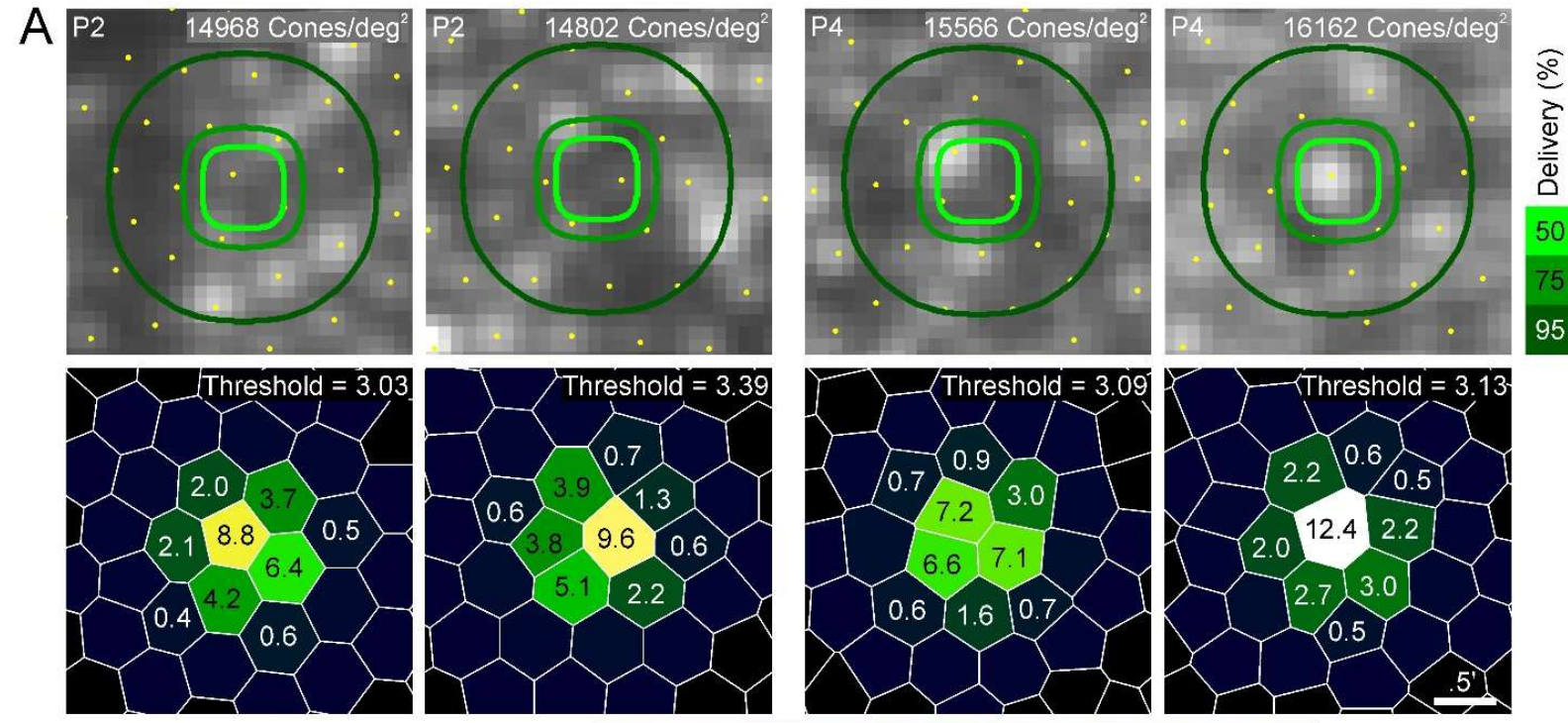

Stimulus light capture (\%)

2

$8,10,12$
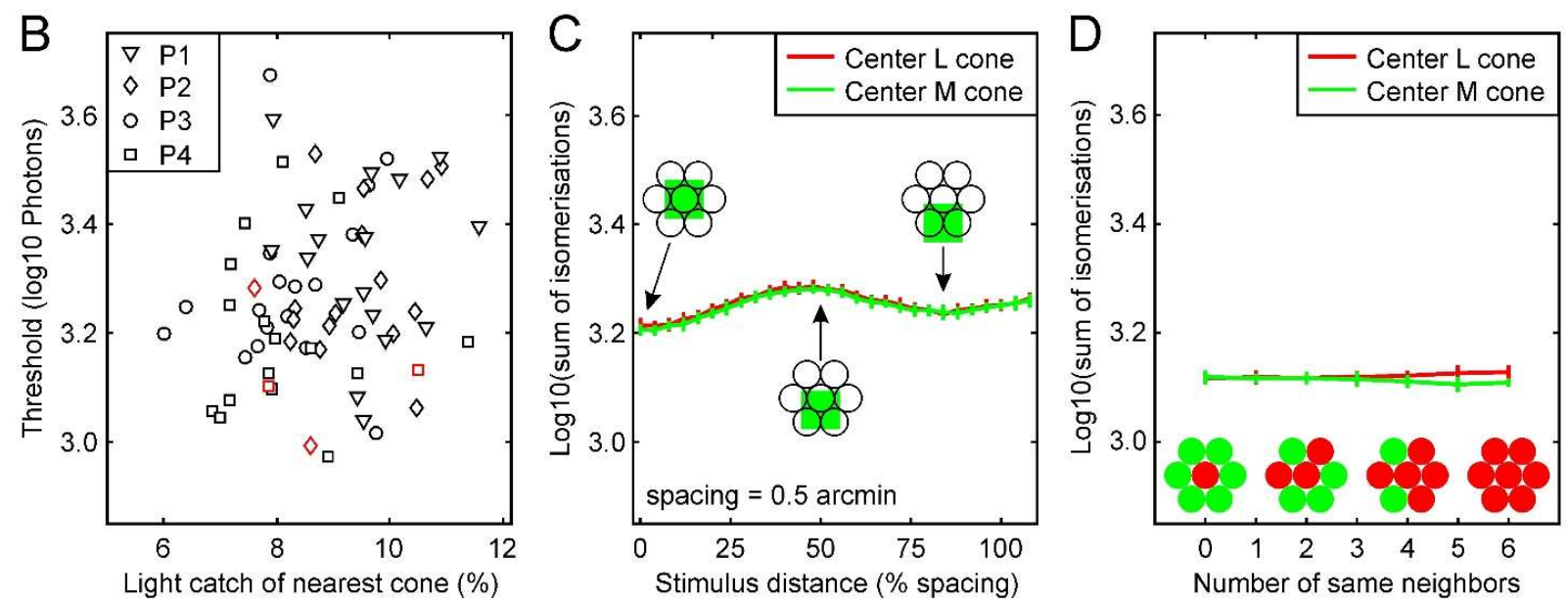

1072 Figure 10: Possible sources of threshold variability. A) We used the cone maps to model the light catch for each cone at the target site. The left two panels show two different test sites of P2, with a similar light catch situation. The observed thresholds for these test sites differed significantly ( $p=0.03$, Mann-Whitney U-test, $n=4)$. The right two panels show two different test sites of $\mathrm{P} 4$, with different light catch situations. In the first condition three cones are supposed to catch the same number of photons, while in the second condition the major portion of stimulus photons are being caught by a single cone. The observed thresholds for these test sites differ insignificantly ( $p=0.49$, Mann-Whitney U-test, $n=4)$. B) Pooling the data across all participants. Small light catch numbers indicate a 3-cone position, high numbers a single cone center position. Example data sets shown in A were highlighted with a red marker outline. There was no significant correlation between observed thresholds and stimulus delivery condition. C) This 
observation was confirmed by an ISETBio model testing the influence of stimulus position on the number of isomerizations. The model showed a maximum change of $0.1 \log 10$ isomerizations for different stimulus positions. D) ISETBio model to test the influence of different cone class compositions at the test site. This model does not contain any inter cone class inhibition and therefore shows only a slight increase of isomerizations if solely L-cones were activated. The error bars in $C$ and $D$ indicate the ISETBio simulated retinal noise.
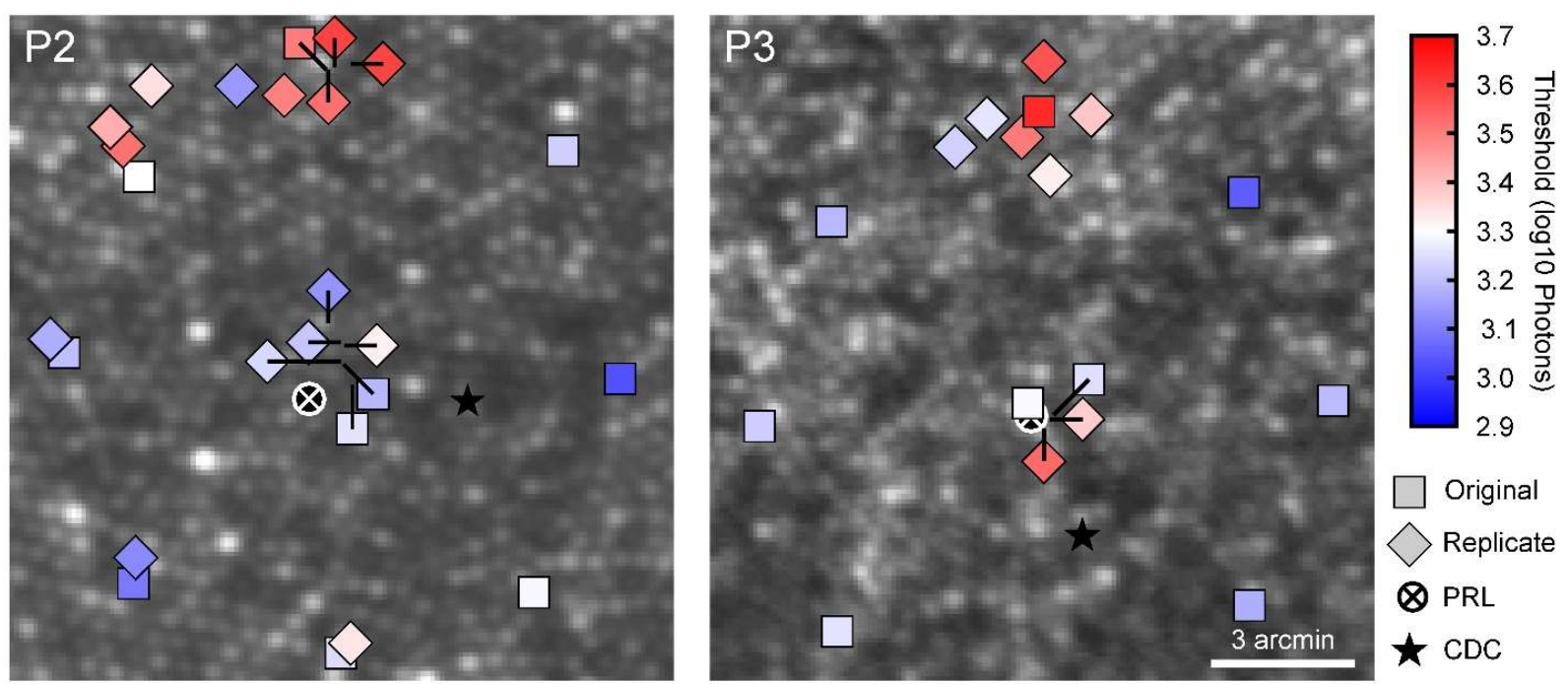

Figure 11: Replicate testing of test sites with conspicuous high thresholds. Squares mark the formerly shown original test sites (Fig. 4), while diamonds mark additionally collected data. The markers match the nominal size of the stimulus. To reduce overlapping, markers were shifted and the tip of a black line indicates the center of the retinal location. For P2 the replicate data points were collected 2 days earlier during a piloting experiment. In P3, the additional locations were tested on the same day, due to the suspiciously high threshold. The PRL is marked with the white circle and crosshair, the cone density centroid by a black star. 